\title{
Pacific
}

Journal of

Mathematics

\section{EXTENSION THEOREMS FOR EXTERNAL CUSPS WITH} MINIMAL REGULARITY

Gabriel Acosta And Ignacio OJEA 


\title{
EXTENSION THEOREMS FOR EXTERNAL CUSPS WITH MINIMAL REGULARITY
}

\author{
GABRIEL ACOSTA AND IGNACIO OJEA
}

\begin{abstract}
Sobolev functions defined on certain simple domains with an isolated singular point (such as power type external cusps) can not be extended in standard, but in appropriate weighted spaces. In this article we show that this result holds for a large class of domains that generalizes external cusps, allowing minimal boundary regularity. The construction of our extension operator is based on a modification of reflection techniques originally developed for dealing with uniform domains. The weight involved in the extension appears as a consequence of the failure of the domain to comply with basic properties of uniform domains, and it turns out to be a quantification of that failure. We show that weighted, rather than standard spaces, can be treated with our approach for weights that are given by a monotonic function either of the distance to the boundary or of the distance to the tip of the cusp.
\end{abstract}

\section{Introduction}

Let $\Omega$ be an open connected set in $\mathbb{R}^{n}$ and let $\omega: \mathbb{R}^{n} \rightarrow \mathbb{R}_{\geq 0}$ be a locally integrable nonnegative function. For $k \in \mathbb{N}$ and $1 \leq p \leq \infty$, the weighted Sobolev space $W_{\omega}^{k, p}(\Omega)\left(W^{k, p}(\Omega)\right.$ if $\left.\omega \equiv 1\right)$ is the space of functions defined on $\Omega$ that have weak derivatives of order $\alpha$, for $|\alpha| \leq k$, and satisfy

$$
\|f\|_{W_{\omega}^{k, p}(\Omega)}:=\sum_{|\alpha| \leq k}\left\|\omega D^{\alpha} f\right\|_{L^{p}(\Omega)}<\infty .
$$

For $\Omega \subsetneq \mathbb{R}^{n}$, an extension operator is a linear bounded operator

$$
\Lambda: W^{k, p}(\Omega) \rightarrow W^{k, p}\left(\mathbb{R}^{n}\right) \quad \text { such that }\left.\Lambda f\right|_{\Omega}=f .
$$

If such an operator exists, then $\Omega$ is called an extension domain for Sobolev spaces (or an EDS).

Supported by ANPCyT under grant number PICT2007-910, and grant number UBACYT EX007. Gabriel Acosta is a member of IMAS-CONICET, and Ignacio Ojea is a fellow of ANPCyT.

MSC2010: 46E35.

Keywords: extension theorems, external cusp, weighted Sobolev spaces. 
It is well known that smooth domains are EDS. In fact, since the boundary of a smooth domain can be locally flattened by means of a regular transformation, $\Lambda$ can be constructed in that case by applying a simple reflection method (see [Adams and Fournier 2003; Maz'ya 2011]). On the other hand, by using the so called Sobolev representation formula in a cone and singular integrals, Calderón [1961] showed that Lipschitz domains are also EDS for $1<p<\infty$. This result was extended to the range $1 \leq p \leq \infty$ by Stein [1970] by using an appropriate averaged reflection procedure.

Reflection type techniques are a natural approach to deal with extension of functions and more complex ways of reflection are needed in order to handle more general domains. In this context Jones [1981] introduced the (broader than Lipschitz) class of $(\varepsilon, \delta)$ domains, also called locally uniform domains and showed, thanks to a subtle reflection method, that every $(\varepsilon, \delta)$ domain is indeed an EDS. In terms of the Whitney decompositions $\mathcal{W}$ and $\mathcal{W}^{c}$ of $\Omega$ and $\left(\Omega^{c}\right)^{o}$, respectively, Jones's idea hinges on the fact that $(\varepsilon, \delta)$ domains enjoy the following properties:

(a) Whitney cubes $Q \in W^{c}$ near $\Omega$ have a "reflected" cube of similar size $Q^{*} \in \mathcal{W}$.

(b) Reflected cubes $Q_{1}^{*}, Q_{2}^{*} \in \mathscr{W}$ of neighboring cubes $Q_{1}, Q_{2} \in W^{c}$ can be joined by a bounded chain of touching cubes in $W$.

Thank to this, an appropriate polynomial approximation of $f$ in $Q^{*}$ can be used to define $\Lambda(f)$ in $Q$.

Even while $(\varepsilon, \delta)$ domains allow very rough boundaries, simple domains such as

$$
\Omega=\left\{x \in \mathbb{R}^{2}: 0<x_{2}<1,\left|x_{1}\right|<x_{2}^{\gamma}\right\}
$$

fail to be EDS for $\gamma>1$. This is a shared feature among general domains having outer peaks. In this regard Maz'ya and Poborchi [1997] introduced:

Definition A. Let $\Omega \subset \mathbb{R}^{n}(n \geq 2)$ be a domain with compact boundary $\partial \Omega$. Assume that $0 \in \partial \Omega$ and that $\partial \Omega \backslash\{0\}$ is locally the graph of a Lipschitz function. We say that $\Omega$ has an external cusp at the origin if there exists a neighborhood of the origin, $U \subset \mathbb{R}^{n}$, such that

$$
U \cap \Omega=\left\{(y, z) \in \mathbb{R}^{n-1} \times \mathbb{R}: y \in \varphi(z) \varpi\right\},
$$

where $\varpi \subset \mathbb{R}^{n-1}$ is a bounded domain and $\varphi: \mathbb{R}^{+} \rightarrow \mathbb{R}^{+}$is a Lipschitz increasing function such that $\varphi(t) / t \rightarrow 0\left(t \rightarrow 0^{+}\right)$and $\varphi(0)=0$.

Remark 1.1. In Definition A, $\varphi$ defines the profile of the cusp; that is, $\Omega$ 's behavior toward the origin is depicted exactly by $\varphi$. Every "horizontal" section of $\Omega$ has the same shape as $\varpi$, scaled by $\varphi$.

For the class of domains given by Maz'ya and Poborchi, Sobolev functions can not be extended in standard, but in weighted spaces. To be more precise: 
Theorem A [Maz'ya and Poborchi 1997; Maz'ya 2011]. Let $\Omega \subset \mathbb{R}^{n}$ be a domain with an external cusp in the origin (in the sense of Definition A).

(a) If $k p<n-1$, or $k=n-1$ and $p=1$, and $\varphi$ satisfies that

$$
\varphi(t) / t \text { is a nondecreasing function, }
$$

then there exists an extension operator

$$
\Lambda: W^{k, p}(\Omega) \rightarrow W_{\sigma}^{k, p}(\mathbb{R}),
$$

where

$$
\sigma(x)=\left\{\begin{array}{cl}
1 & \text { if } x \in \Omega, \\
\left(\frac{\varphi(|x|)}{|x|}\right)^{k} & \text { if } x \in \Omega^{c} .
\end{array}\right.
$$

(b) If $k p>n-1$, and $\varphi$ is such that

$$
\text { there exists } C_{\varphi} \text { constant such that } \varphi(2 t) \leq C_{\varphi} \varphi(t) \text {, }
$$

then there exists an extension operator

$$
\Lambda: W^{k, p}(\Omega) \rightarrow W_{\sigma}^{k, p}(\mathbb{R}),
$$

where

$$
\sigma(x)=\left\{\begin{array}{cc}
1 & \text { if } x \in \Omega, \\
\left(\frac{\varphi(|x|)}{|x|}\right)^{(n-1) / p} & \text { if } x \in \Omega^{c} .
\end{array}\right.
$$

(c) In either case (a) or (b), assuming (1-4) holds, if $\tilde{\sigma}$ is such that there is an extension operator $\tilde{\Lambda}: W^{k, p}(\Omega) \rightarrow W_{\tilde{\sigma}}^{k, p}\left(\mathbb{R}^{n}\right)$, then

$$
\tilde{\sigma}(x) \leq C \sigma(x) \text { for all } x \in U \backslash \Omega \text {. }
$$

Remark 1.2. Under the same hypotheses of Theorem A, and for the critical case $k p=n-1$, with $\varphi$ satisfying a condition similar to (1-4) (but slightly weaker), the authors show that there exists an extension operator

$$
\Lambda: W^{k, p}(\Omega) \rightarrow W_{\sigma}^{k, p}(\mathbb{R}),
$$

where

$$
\sigma(x)=\left(\frac{\varphi(|x|)}{|x|}\right)^{k}\left|\log \frac{\varphi(|x|)}{|x|}\right|^{1-\frac{1}{p}} .
$$

Observe that property (1-4) excludes exponential cusps with profiles given by functions such as $\varphi(t)=a e^{-b / t}$. This property is not necessary for the existence of the extension operator in the case $k p<n-1$, but it is used in the proof of the optimality of the weight $\sigma$ (item (c)). Throughout this work the weight given in item (a) (respectively (b)) is called derivative (respectively dimensional) weight. 
Among several applications [Acosta et al. 2007; Acosta and Armentano 2011], Theorem A allows us to handle convergence issues in the context of finite element approximations of elliptic partial differential equations. External cusps can not be exactly fitted by polygonal discretizations and the finite element mesh needs to be graded according to $\sigma(x)$ in order to get optimal order error estimates. On the other hand, weighted Sobolev spaces in external cusps are of interest in linear elasticity. Indeed, the classical Korn inequality, fundamental to proving existence of solutions of the linearized elasticity equations, does not hold in domains with outer peaks, but a variant involving weights depending on the distance to the boundary or the distance to the tip of the cusp does hold. Similar results hold for the divergence operator for which a continuous right inverse can be defined on this kind of weighted spaces [Acosta et al. 2006; 2012; Durán and López García 2010a; 2010b]. In this context it is clear that a version of Theorem A for weights of the type described can also be useful in applications.

In this paper we present a twofold generalization of Theorem A: a first involving a broader class of domains and a second allowing weighted spaces. Concerning the former, and loosely speaking, we prove, in terms of Maz'ya and Poborchi's definition, that the extension can be performed even in a context in which $\varphi$ does not give the precise profile of $\Omega$, but just an approximate description of the narrowing toward the origin, as long as the boundary of $\Omega$ satisfies minimal regularity conditions. We also show that the extension is possible with either the derivative or the dimensional weight regardless the relationship between the parameters $k, p$ and $n$ (although we do not treat the critical case $k p=n-1$ ). On the other hand we also present some results for the weighted case. Even while Chua [1992; 1994] has shown that Jones's technique can also be applied to handle extensions in $W_{\omega}^{k, p}$ for very general weights (essentially if $\omega$ is doubling and if functions in $W_{\omega}^{k, p}$ can be well approximated in cubes by polynomials), in our context, for several reasons pointed out later, we need to restrict our study to weights that are mainly given by monotonic functions of the distance to the boundary or the distance to the tip of the cusp.

Our arguments can be summarized as follows. We introduce a general definition of external cusps, that includes those domains satisfying Definition A (see Definitions 2.6 and 2.7). Our definition is given in terms of (a) a representative chain (called the spine of the cusp) of central cubes belonging to $\mathcal{W}$ and (b) certain local variable uniformity property. The latter property ensures that any cube $Q \in W^{c}$ close enough to $\Omega$ has a reflected cube $Q^{*} \in \mathcal{W}$ of the type needed in Jones's arguments. Therefore, in a first stage, a local unweighted extension $\grave{a}$ la Jones is carried out. In a second stage, and for cubes $T \in W^{c}$ that are not too close to $\Omega$, we define a reflected set $T^{*}$ that is not necessarily a single, but a finite union of cubes belonging to the central spine. We show that $T^{*}$ can be essentially taken 
in two different ways, where either the cardinal of $T^{*}$ is large and the size of the cubes in $T^{*}$ is much smaller than that of $T$, or $T^{*}$ has a single cube of the same size of $T$ but the distance from $T$ to $T^{*}$ is much larger than the size of $T$. In each case our arguments lead to a proper weight required in the extension process. Our reflection procedure makes the treatment of the weighted case easy, for weights of the type described before.

The paper is organized in the following way. In Section 2 we introduce some notation, recall basic aspects of uniform domains and Whitney decompositions, and finally give our definition of normal and curved cusps. Section 3 is devoted to proving some preliminary lemmas on polynomials. In Section 4 we present a detailed proof of the extension theorem for normal cusps (Theorem 4.1) and the adaptation for curved cusps is developed in Section 5 (Theorem 5.1). Section 6 is just an appendix to Sections 4 and 5, where some accessory results are proved. In Section 7 we treat the weighted case. Finally, in Section 8 we summarize the results presented in the paper, add some comments, and show a few examples.

\section{A general definition of external cusps}

Given a rectangle $R \subset \mathbb{R}^{n}$ with edges parallels to the coordinate axis (this is the case for all the rectangles considered in this article), the size vector of $R$ is denoted by $\vec{\ell}(R)=\left(\ell_{1}(R), \ell_{2}(R), \ldots, \ell_{n}(R)\right)$, where $\ell_{i}(R)$ is the length of $R$ 's $i$-th edge. For a cube $Q$ we use $\ell(Q)$ to denote the length of any of its edges, and for a rectangle $R$, we define $\ell(R):=\max _{1 \leq i \leq n}\left\{\ell_{i}(R)\right\}$. We say that two rectangles $R_{1}$, $R_{2}$ are equivalent, and write $R_{1} \sim R_{2}$, if there are constants $C_{1}, C_{2}$ such that

$$
C_{1} \ell_{i}\left(R_{1}\right) \leq \ell_{i}\left(R_{2}\right) \leq C_{2} \ell_{i}\left(R_{1}\right)
$$

for $1 \leq i \leq n$. For a rectangle $R$, we denote its center by $c_{R}$. If $c_{R}=\left(c_{1}, \ldots, c_{n}\right)$, the upper face $F_{R}^{u}$ of $R$ is given by

$$
F_{R}^{u}=\left\{\left(x_{1}, \ldots, x_{n}\right) \in R: x_{n}=c_{n}+\frac{1}{2} \ell_{n}(R)\right\},
$$

and the lower face $F_{R}^{l}$ is defined analogously.

Given a rectangle $R$, we denote by $a R(a>1)$ the expanded rectangle centered at $c_{R}$ with edges $\ell_{i}(a R)=a \ell_{i}(R)$. For a cube $Q$, centered at $c_{Q}=\left(c_{1}, \ldots, c_{n}\right)$ we set $z_{Q}=c_{n}-\frac{1}{2} \ell(Q)$ (the last coordinate of points belonging to $F_{Q}^{l}$ ). We say that $R_{1}$ and $R_{2}$ are touching rectangles if $R_{1}^{o} \cap R_{2}^{o}=\varnothing$ and $R_{1} \cap R_{2}=F$ with $F$ a face of $R_{1}$ or $R_{2}$.

For every collection of sets $\mathscr{C}$, we denote by $\bigcup \mathscr{C}=\bigcup_{S \in \mathscr{C}} S$ the union of all the sets in $\mathscr{C}$. Finally, throughout this article $\hat{x}_{n}$ stands for the $x_{n}$ axis, and $C$ denotes a generic constant that may change from line to line.

Let us recall a definition that plays a crucial role in the sequel. 
Definition 2.1 (locally uniform domains). $D$ is an $(\varepsilon, \delta)$ domain if for all $x, y \in D$ with $|x-y|<\delta$ there is a rectifiable curve $\gamma$ joining $x$ and $y$ such that

$$
\begin{aligned}
\ell(\gamma) & <\frac{|x-y|}{\varepsilon}, \\
d_{\partial \Omega}(z) & >\frac{\varepsilon|x-z||z-y|}{|x-y|} \quad \text { for all } z \in \gamma .
\end{aligned}
$$

If $\delta>\operatorname{diam} D$, we say that $D$ is a uniform domain (note that then $D$ is connected).

Lemma 2.2 [Jones 1981]. If $D$ is $a(\varepsilon, \delta)$ domain, then $|\partial D|=0$.

Uniform domains include Lipschitz domains, but they form a much larger class. If fact, if $\Omega$ is uniform, $\partial \Omega$ could be very rough. On the other hand, classical examples of domains with a single singular point that are not uniform are precisely external cusps of power type, where property (2-2) fails, and also inner cups of the same kind, where property (2-1) fails.

Below we define cusps in terms of its Whitney decomposition, so we recall:

Theorem 2.3 (Whitney). Let $\Omega \subset \mathbb{R}^{n}, \Omega \neq \mathbb{R}^{n}$, be an open set. Then there is a collection $\mathscr{W}=\mathscr{W}(\Omega):=\left\{Q_{j}\right\}$ of (countably) infinite dyadic closed cubes such that $\Omega=\bigcup W$, and

- $Q_{j}^{o} \cap Q_{k}^{o}=\varnothing$ for all $Q_{j}, Q_{k} \in W(\Omega)$,

- $\ell\left(Q_{j}\right) \leq d\left(Q_{j}, \partial \Omega\right) \leq 4 \sqrt{n} \ell\left(Q_{j}\right)$ for all $Q_{j}$,

- if $Q_{j} \cap Q_{k} \neq \varnothing$, then $\ell\left(Q_{j}\right) \leq 4 \ell\left(Q_{k}\right)$.

Remark 2.4. The proof of Theorem 2.3 can be found, for instance, in [Stein 1970]. One can easily observe that for any pair of open sets $A$ and $B$ with $A \subset B$, every cube $Q \in \mathcal{W}(A)$ is contained in some cube $\widetilde{Q} \in \mathcal{W}(B)$.

Remark 2.5. Like in Definition A, the cusps defined below are assumed to be tangential to a certain fixed direction that is taken arbitrarily along $\hat{x}_{n}$, with the tip of the cusp placed at the origin 0 . Our definition is "intrinsic" to some extent, since it is based on Whitney cubes and a certain variable uniformity property.

Let us recall that we denote by $\mathcal{W}$ and $\mathscr{W}^{c}$ the Whitney decompositions of $\Omega$ and $\left(\Omega^{\mathrm{c}}\right)^{\mathrm{o}}$, respectively.

Definition 2.6 (normal cusp). Let $\Omega \subset \mathbb{R}^{n}$ be an open set such that $0 \in \partial \Omega$. Let $\varepsilon>0$ and $K>1$ be given parameters. We say that $\Omega$ has a $(\varepsilon, K)$-normal external cusp (or outer peak) at the origin if it satisfies:

(i) There exists a chain $\mathscr{S}=\left\{S_{i}\right\}_{i=1}^{\infty} \subset \mathcal{W}$ of cubes increasingly numbered towards the origin $\left(d\left(S_{i+1}, 0\right) \leq d\left(S_{i}, 0\right)\right)$, such that

$$
\begin{gathered}
S_{i} \cap S_{i+1}=F_{S_{i+1}^{u}}^{u} \\
d\left(S_{i}, 0\right) \rightarrow 0 \quad(i \rightarrow \infty) .
\end{gathered}
$$


(ii) Using the abbreviated notation $z_{i}:=z_{S_{i}}$ for cubes $S_{i} \in \mathscr{T}$, denoting by $S(z)$ the cube at height $z>0$ (i.e., $S(z):=S_{i}$ if $z_{i} \leq z<z_{i-1}$ ) and by $i_{z}$ the index of the cube at height $z$ (i.e., $i_{z}=i$ if $S_{i}=S(z)$ ), we have that

$$
\left\{x \in \Omega: x_{n}<z\right\} \subset \bigcup_{i=i_{z}}^{\infty} \Omega_{i} \text { for any } z_{1}>z>0,
$$

where $\Omega_{i}=K S_{i} \cap \Omega$.

(iii) For every pair of points $x, y \in \Omega_{i} \cup \Omega_{i+1}$, there is a rectifiable curve $\gamma \subset \Omega$, joining $x$ and $y$, that satisfies

(iv) We have

$$
\begin{aligned}
\ell(\gamma) & \leq \frac{1}{\varepsilon}|x-y|, \\
d_{\partial \Omega}(z) & \geq \varepsilon \frac{|x-z||z-y|}{|x-y|} .
\end{aligned}
$$

The set $\mathscr{Y}$ is named the spine of $\Omega$.

Condition (2-3) means that the spine $\mathscr{Y}$ is straight, and parallel to $\hat{x}_{n}$. It also implies that the chain is decreasing, or $\ell\left(S_{i+1}\right) \leq \ell\left(S_{i}\right)$. This last fact is not really necessary but is assumed for the sake of simplicity: the sizes of the cubes in $\mathscr{Y}$ could oscillate, as long as its oscillation is controlled by some universal parameter, depending only on $\Omega$.

On the other hand, conditions (2-3) and (2-4) imply that every cube $S_{i}$ of $\mathscr{S}$ touches $\hat{x}_{n}$, while (2-5) guarantees that $\Omega$ 's behavior (its narrowing toward the origin) is faithfully represented by the behavior of the chain $\mathscr{S}$ : a fixed expansion of the tails of $\mathscr{Y}$ reaches the whole boundary of $\Omega$ below certain height $z$, and consequently $\partial \Omega$ narrows toward the origin as fast as $\ell(S(z))$. In other words, the function $\ell(S(z))$ plays the role of $\varphi(z)$ in Definition A.

Finally, conditions (2-6) and (2-7) provide some regularity to the boundary of $\Omega$ and exclude the existence of non connected components in $\Omega$.

Condition (2-8) is not necessary at all for the extension process. We include it in order to exclude cones and other nonsingular domains from our definition of a cusp. However, it is important to notice that our extension theorems (see Theorems 4.1 and 5.1) hold even for domains on which (2-8) is not fulfilled. In that case the weight turns to be a constant, and a classical (unweighted) extension is obtained.

Normal cusps are, somehow, "symmetric" with respect to $\hat{x}_{n}$. More precisely, normal cusps are those that grow around an axis that is placed approximately at its center; see Figure 1. The following definition includes cusps that are tangential to a certain axis, which is not necessarily interior to the domain. 

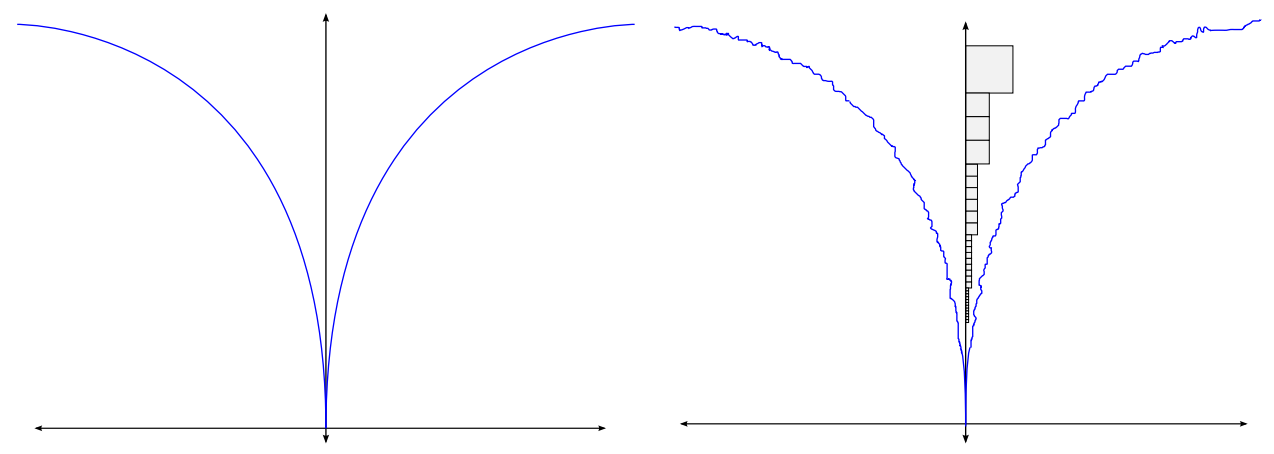

Figure 1. Cusp of type (1-2) versus normal cusp with its spine.

Definition 2.7 (curved cusp). Let $\Omega \subset \mathbb{R}^{n}$ be an open set such that $0 \in \partial \Omega$. Let $\varepsilon>0$ and $K>1$ be given parameters. We say that $\Omega$ has an $(\varepsilon, K)$-curved external cusp (or outer peak) at the origin if there exists a chain of cubes $\mathscr{Y}=\left\{S_{i}\right\}_{i}, S_{i} \in \mathcal{W}$, increasingly numbered toward the origin 0 , satisfying

$$
\begin{gathered}
S_{i} \cap S_{i+1} \neq \varnothing, \\
d\left(S_{i}, \hat{x_{n}}\right) \leq C_{\Omega} \ell\left(S_{i}\right) \text { for some } C_{\Omega}, \\
\ell\left(S_{i+1}\right) \leq \ell\left(S_{i}\right),
\end{gathered}
$$

and if $\Omega$ satisfies conditions (ii), (iii) and (iv) of Definition 2.6.

Conditions (2-9) and (2-10) constitute a relaxation of condition (2-3). Here, the chain is not forced to be straight and parallel to $\hat{x}_{n}$, but to approximate it asymptotically. As we remarked earlier, condition (2-11) is not necessary, but comfortable. Since we abandoned property (2-3), (2-11) is not implicit any more, and so we include it in the definition of curved cusps.

Remark 2.8. For both normal or curved cusps we add two extra conditions that are analogous to the ones required by Maz'ya and Poborchi in Theorem A:

$$
\begin{aligned}
\frac{\ell\left(S_{i}\right)}{z_{i}} \leq C \frac{\ell\left(S_{j}\right)}{z_{j}} & \text { for all } i>j, C \text { constant. } \\
\ell\left(S_{j}\right) \leq K \ell\left(S_{i}\right) & \text { for all } i>j \text { such that } d\left(S_{i}, 0\right)>\frac{1}{2} d\left(S_{j}, 0\right) .
\end{aligned}
$$

Property (2-12) is a generalization of (1-3). We use it to prove item (a) in Theorems 4.1 and 5.1. On the other hand, (2-13) is a generalization of (1-4), and it is necessary for the proof of item (b) in both theorems.

Finally, let us point out some details that arise from the comparison between Definition A and Definitions 2.6 and 2.7. 
Note that a set satisfying Definition A is a domain that, being cut at different heights, shows the same shape, scaled according to the height by the function $\varphi$. This is clearly not the case in Definition 2.7. On the other hand, since the decreasing conditions are stated on the spine $\mathscr{Y}$, and not on $\partial \Omega$, our definitions allow small oscillations in $\partial \Omega$ instead of the monotonic behavior imposed by $\varphi$. Furthermore, Definition A asks $\partial \Omega \backslash\{0\}$ to be locally Lipschitz, whereas the variable uniform properties (2-6) and (2-7) constitute a much more relaxed condition. In Section 8 we provide some examples that show in different ways the generalization implicit in Definitions 2.6 and 2.7 .

\section{Some preliminary lemmas}

For a polynomial $P, \operatorname{deg} P$ stands for the degree of $P$.

Lemma 3.1. Let $R$ be a rectangle, $P$ a polynomial with $\operatorname{deg} P \leq k$, then

$$
\|P\|_{L^{\infty}(R)} \leq \frac{C}{|R|^{1 / p}}\|P\|_{L^{p}(R)}, \quad 1 \leq p \leq \infty,
$$

with $C$ depending only on $k$.

Proof. Let $\widehat{Q}=[-1 / 2,1 / 2]^{n}$. Let $F: \widehat{Q} \rightarrow R$ be the linear application: $F: \hat{x} \rightarrow x$, $F(\hat{x})=\vec{\ell}(R) \cdot \hat{x}^{t}+c_{R}$. Observe that $|D F|=|R|$. We consider another polynomial $\widehat{P}$ defined on $\widehat{Q}$ by $\widehat{P}(\hat{x})=P(F(\hat{x}))$. Notice that $\operatorname{deg} \widehat{P}=\operatorname{deg} P$. Changing variables, we obtain

$$
\begin{aligned}
\|P\|_{L^{\infty}(R)} & =\|\widehat{P}\|_{L^{\infty}(\widehat{Q})} \leq \widehat{C}\|\widehat{P}\|_{L^{p}(\widehat{Q})} \\
& =\widehat{C}\left(\int_{\hat{Q}}|\widehat{P}(\hat{x})|^{p} d \hat{x}\right)^{1 / p} \leq \widehat{C}\left(\int_{R}|P(x)|^{p} \frac{1}{|R|} d x\right)^{1 / p},
\end{aligned}
$$

where the first inequality follows from the equivalence of norms on the finite dimensional space of polynomials of degree $\leq k$ defined on $\widehat{Q}$.

Lemma 3.2. Let $R$ and $Q$ be rectangles such that $R \subset Q$, and $P$ a polynomial with $\operatorname{deg} P \leq k$. Then there exists a constant $C$, depending only on $k$, such that

$$
\|P\|_{L^{p}(Q)} \leq C\left(\frac{|Q|}{|R|}\right)^{1 / p} \sum_{|\alpha| \leq k}\left\|D^{\alpha} P\right\|_{L^{p}(R)} \vec{\ell}(Q)^{\alpha} .
$$

Proof. We may assume that $0 \in R$. Let $q \in Q$ be such that $\|P\|_{L^{\infty}(Q)}=|P(q)|$, then

$$
\begin{aligned}
\|P\|_{L^{p}(Q) \leq\|P\|_{L^{\infty}(Q)}|Q|^{1 / p}} & =|P(q) \| Q|^{1 / p} \leq|Q|^{1 / p} \sum_{|\alpha| \leq k}\left|D^{\alpha} P(0)\right| \frac{\left|q^{\alpha}\right|}{\alpha !} \\
& \leq C|Q|^{1 / p} \sum_{|\alpha| \leq k}\left\|D^{\alpha} P\right\|_{L^{\infty}(R)} \vec{\ell}(Q)^{\alpha} \\
& \leq C\left(\frac{|Q|}{|R|}\right)^{1 / p} \sum_{|\alpha| \leq k}\left\|D^{\alpha} P\right\|_{L^{p}(R)} \vec{\ell}(Q)^{\alpha} .
\end{aligned}
$$


Corollary 3.3. Let $R \subset Q$ be rectangles such that $|Q| /|R| \leq C$, and $P$ a polynomial with $\operatorname{deg} P \leq k$. Then there exists a constant $C$, depending only on $k$, such that

$$
\|P\|_{L^{p}(Q)} \leq C\|P\|_{L^{p}(R)} .
$$

Remark 3.4. A version of Corollary 3.3 is proved in [Jones 1981, Lemma 2.1]. In our case we need to compare polynomials in rectangles that are not of similar size (a fact that eventually leads to the weights involved in the extension) and we need the less comfortable variant given in Lemma 3.2.

Following Jones [1981], the extension operator is built in terms of polynomials that approximate a function in certain sets. Let $f \in W^{k, p}(\Omega)$, and $S \subset \Omega$ a set of positive measure, we denote by $P_{k-1}(S)$ (or just $P(S)$ if the degree is clear from the context) the unique polynomial of degree $k-1$ such that

$$
\int_{S} D^{\alpha}\left(f-P_{k-1}(S)\right)=0 \quad \text { for all } \alpha \text { with }|\alpha| \leq k-1 .
$$

Thanks to the Poincaré inequality one knows that $P(R)$ has good approximation properties if $R$ is a rectangle (regardless of the eccentricity of $R$ ). In the spirit of Lemma 2.2 in [Jones 1981] we also need such a result for the union of two touching rectangles of similar size.

Lemma 3.5. Let $R_{1}, R_{2}$ be rectangles such that $R_{1} \sim R_{2}$. Assume that either $R_{1}$ and $R_{2}$ are touching or that $R_{1} \subseteq R_{2}$ (renumbering if necessary). Then, for any $f$ in $W^{k, p}\left(R_{1} \cup R_{2}\right)$,

$$
\left\|f-P\left(R_{1} \cup R_{2}\right)\right\|_{L^{p}\left(R_{1} \cup R_{2}\right)} \leq C \ell\left(R_{1}\right)^{k} \sum_{|\alpha|=k}\left\|D^{\alpha} f\right\|_{L^{p}\left(R_{1} \cup R_{2}\right)} .
$$

Proof. Clearly it is enough to prove the result in the case $k=1$. If $R_{1} \subseteq R_{2}$ (or vice versa) then the result follows by the Poincare inequality for convex domains (or from a fixed cube by scaling arguments). Let us then treat the case of touching rectangles. Define $f_{R_{1} \cup R_{2}}=\frac{1}{\left|R_{1} \cup R_{2}\right|} \int_{R_{1} \cup R_{2}} f$; then $P\left(R_{1} \cup R_{2}\right)=f_{R_{1} \cup R_{2}}$. Write $\left\|f-P\left(R_{1} \cup R_{2}\right)\right\|_{L^{p}\left(R_{1} \cup R_{2}\right)}^{p}=\left\|f-P\left(R_{1} \cup R_{2}\right)\right\|_{L^{p}\left(R_{1}\right)}^{p}+\left\|f-P\left(R_{1} \cup R_{2}\right)\right\|_{L^{p}\left(R_{2}\right)}^{p}$. We now show how to deal with the first term (the other follows similarly). We have $\left\|f-P\left(R_{1} \cup R_{2}\right)\right\|_{L^{p}\left(R_{1}\right)}$
\[ \frac{\left|R_{1}\right|}{\left|R_{1}\right|+\left|R_{2}\right|}\left\|f-P\left(R_{1}\right)\right\|_{L^{p}\left(R_{1}\right)}+\frac{\left|R_{2}\right|}{\left|R_{1}\right|+\left|R_{2}\right|}\left\|f-P\left(R_{2}\right)\right\|_{L^{p}\left(R_{1}\right)}, \] and the first term is fine. For the other term we write

$$
\left\|f-P\left(R_{2}\right)\right\|_{L^{p}\left(R_{1}\right)} \leq\left\|f-P\left(R_{1}\right)\right\|_{L^{p}\left(R_{1}\right)}+\left\|P\left(R_{1}\right)-P\left(R_{2}\right)\right\|_{L^{p}\left(R_{1}\right)},
$$

and again the first term is all right. In order to treat $\left\|P\left(R_{1}\right)-P\left(R_{2}\right)\right\|_{L^{p}\left(R_{1}\right)}$ observe 
that since $R_{1}$ and $R_{2}$ are touching, there exist rectangles $R_{3}$ and $R_{4}$ such that $R_{3} \subset R_{1} \cup R_{2} \subset R_{4}$ and

$$
R_{1} \sim R_{1} \cap R_{3} \sim R_{2} \cap R_{3} \sim R_{2} \sim R_{3} \sim R_{4} .
$$

Then (using, for instance, Corollary 3.3)

$\left\|P\left(R_{1}\right)-P\left(R_{2}\right)\right\|_{L^{p}\left(R_{1}\right)} \leq C\left\|P\left(R_{1}\right)-P\left(R_{3}\right)\right\|_{L^{p}\left(R_{1} \cap R_{3}\right)}+\left\|P\left(R_{3}\right)-P\left(R_{2}\right)\right\|_{L^{p}\left(R_{4}\right)}$ and

$$
\left\|P\left(R_{1}\right)-P\left(R_{3}\right)\right\|_{L^{p}\left(R_{1} \cap R_{3}\right)} \leq\left\|P\left(R_{1}\right)-f\right\|_{L^{p}\left(R_{1}\right)}+\left\|f-P\left(R_{3}\right)\right\|_{L^{p}\left(R_{3}\right)},
$$

while (using again Corollary 3.3)

$$
\begin{aligned}
\left\|P\left(R_{3}\right)-P\left(R_{2}\right)\right\|_{L^{p}\left(R_{4}\right)} & \leq C\left\|P\left(R_{3}\right)-P\left(R_{2}\right)\right\|_{L^{p}\left(R_{2} \cap R_{3}\right)} \\
& \leq C\left\|f-P\left(R_{2}\right)\right\|_{L^{p}\left(R_{2}\right)}+\left\|P\left(R_{3}\right)-f\right\|_{L^{p}\left(R_{3}\right)} .
\end{aligned}
$$

The lemma follows.

\section{Extension}

The objective of this section is to build an extension operator for cusps in the unweighted case $W^{k, p}$, proving the main result of this paper.

Theorem 4.1. Let $\Omega \subset \mathbb{R}^{n}$ be a domain with an external normal cusp at the origin.

(a) If the spine 9 satisfies (2-12), there is an extension operator

$$
\Lambda: W^{k, p}(\Omega) \rightarrow W_{\sigma}^{k, p}\left(\mathbb{R}^{n}\right),
$$

where

$$
\sigma(x)=\left\{\begin{array}{cl}
1 & \text { if } x \in \Omega, \\
\left(\frac{\ell(S(|x|))}{|x|}\right)^{k} & \text { if } x \in \Omega^{c} .
\end{array}\right.
$$

(b) If the spine $\mathscr{Y}$ satisfies (2-13), there is an extension operator

$$
\Lambda: W^{k, p}(\Omega) \rightarrow W_{\sigma}^{k, p}\left(\mathbb{R}^{n}\right),
$$

where

$$
\sigma(x)=\left\{\begin{array}{cl}
1 & \text { if } x \in \Omega, \\
\left(\frac{\ell(S(|x|))}{|x|}\right)^{(n-1) / p} & \text { if } x \in \Omega^{c} .
\end{array}\right.
$$

(c) In either case (a) or (b), assuming (2-13) holds, if $\tilde{\sigma}$ is such that there is an extension operator $\tilde{\Lambda}: W^{k, p}(\Omega) \rightarrow W_{\tilde{\sigma}}^{k, p}\left(\mathbb{R}^{n}\right)$, then

$$
\tilde{\sigma}(x) \leq C \sigma(x) \text { for all } x \in U \backslash \Omega \text {. }
$$


Remark 4.2. Theorem 4.1 states the existence of a general extension operator for normal cusps. The only difference between items (a) and (b) is the requirement of properties (2-12) and (2-13) respectively. In contrast to Theorem A, no conditions on $k, p$ or $n$ are needed in the proof of any of these cases.

Remark 4.3. Condition (2-12) is not necessary if $k p \neq 1$ (see Remark 4.20). Consequently, since every domain satisfying Definition A is an external cusp (normal or curved) in our terms (see Corollary 8.6), hypothesis (1-3) is not necessary in Theorem A when $k p \neq 1$.

Below we provide a detailed proof for items (a) and (b) of Theorem 4.1. Item (c) is discussed in the last section of this article. The case of curved cusps requires a little modification (similar to that needed in [Maz'ya and Poborchi 1997]) of our arguments and is therefore sketched later in Theorem 5.1.

Let us notice that thanks to item (iii) in Definition 2.6 and the results of Jones for locally uniform domains, it is clear that it is enough to construct an extension operator $\Lambda$ for functions $u$ such that $\operatorname{supp}(u) \subset D_{r}=\left\{x=\left(x_{1}, \ldots, x_{n}\right):\left|x_{n}\right|<r / 2\right\}$, where $r \ll \sum_{i=1}^{\infty} \ell\left(S_{i}\right)$. Our operator $\Lambda$ is defined on a set of cubes belonging to $\mathscr{W}^{c}$. Let us call $\mathscr{W}_{2} \subset \mathscr{W}^{c}$ the set of cubes that belong to $\mathscr{W}^{c}$ and are contained in $D_{r}$. We divide $\mathcal{W}_{2}$ into three parts related to three different stages of the extension process:

$$
\begin{aligned}
& \mathscr{W}_{3}=\left\{Q \in W_{2}: z_{Q}>0 \text { and } \ell(Q) \leq\left(\frac{\varepsilon}{5 \sqrt{n}} \frac{K-1}{K}\right) \ell\left(S\left(z_{Q}\right)\right)\right\}, \\
& \mathcal{W}_{4}=\left\{Q \in \mathscr{W}_{2} \backslash \mathcal{W}_{3}: z_{Q}>0 \text { and } \ell(Q) \leq z_{Q} /(8 \sqrt{N})\right\}, \\
& \mathscr{W}_{5}=\left\{Q \in \mathscr{W}_{2} \backslash\left(\mathcal{W}_{3} \cup \mathcal{W}_{4}\right)\right\} .
\end{aligned}
$$

Furthermore, let us denote by $Q_{j}$ the cubes in $\mathscr{W}_{3}$, so $\mathscr{W}_{3}=\left\{Q_{j}\right\}_{j}$, and similarly by $\left\{T_{j}\right\}_{j}$ the cubes in $\mathscr{W}_{4}$ and by $\left\{U_{j}\right\}_{j}$ those in $\mathscr{W}_{5}$. Finally, let $\left\{\xi_{j}\right\}_{j},\left\{\varphi_{j}\right\}_{j}$ and $\left\{\psi_{j}\right\}_{j}$ be a partition of unity on $\bigcup \mathcal{W}_{2}$, such that $\xi_{j} \in C_{0}^{\infty}, \varphi_{j} \in C_{0}^{\infty}, \psi_{j} \in C_{0}^{\infty}$; $\operatorname{sop}\left(\xi_{j}\right) \subset \frac{17}{16} Q_{j}, \operatorname{sop}\left(\varphi_{j}\right) \subset \frac{17}{16} T_{j}, \operatorname{sop}\left(\psi_{j}\right) \subset \frac{17}{16} U_{j}$, and

$$
\sum_{j} \xi_{j}(x)+\sum_{j} \varphi_{j}(x)+\sum_{j} \psi_{j}(x)=1 \quad \text { for all } x \in \bigcup W_{2} \text {. }
$$

As usual, we may also assume that

$$
\left|D^{\alpha} \xi_{j}(x)\right| \leq \frac{C}{\ell\left(Q_{j}\right)^{|\alpha|}}, \quad\left|D^{\alpha} \varphi_{j}(x)\right| \leq \frac{C}{\ell\left(T_{j}\right)^{|\alpha|}}, \quad\left|D^{\alpha} \psi_{j}(x)\right| \leq \frac{C}{\ell\left(U_{j}\right)^{|\alpha|}} .
$$

At each stage of the extension process we define a polynomial for each cube in each set $\mathscr{W}_{i}$ (in the first stage, a polynomial for each cube in $W_{3}$, and so on). The extension operator is finally constructed by using our partition of the unity.

Following Jones's ideas we define, for each $Q \in \mathcal{W}_{2}$, some set $S(Q) \subset \Omega$, so the polynomial for $Q$ will be $P(S(Q))$. 
First stage. This stage closely follows the reflection method given in [Jones 1981]. It is based on Lemma 4.6, where the existence of a reflected cube for every $Q \in \mathcal{W}_{3}$ is proved. We need to state a previous lemma.

Lemma 4.4. Given $\Omega$ an external normal cusp with parameters $\varepsilon, K$, there is a constant $\widetilde{K}$ (that could be taken to be $\widetilde{K}=K(K+1) / 2$ ) such that if $x \in \Omega$ and

$$
z_{i}-\frac{K-1}{2} \ell\left(S_{i}\right) \leq x_{n} \leq z_{i}+\frac{K+1}{2} \ell\left(S_{i}\right)
$$

then $x \in \widetilde{K} S_{i}$.

Proof. Let us take $j=i_{x_{n}}$. We suppose $j<i$ (the complementary case is analogous). Property (2-5) implies that $K S_{j} \ni x$. On the other hand $z_{j} \leq z_{i}+\frac{K+1}{2} \ell\left(S_{i}\right)$. But, since $\ell\left(S_{j}\right) \geq \ell\left(S_{i}\right)$, we have $\ell\left(S_{j}\right)=2^{N} \ell\left(S_{i}\right)$ for some $N \in \mathbb{N}_{0}$. The largest size of $S_{j}$ is obtained when the cubes in $\mathscr{S}$ grow exponentially between $S_{i}$ and $S_{j}$. In that case,

$$
z_{j}-z_{i}=\sum_{m=0}^{N-1} 2^{m} \ell\left(S_{i}\right) \leq \frac{K+1}{2} \ell\left(S_{i}\right)
$$

and $2^{N} \leq(K+1) / 2$, which leads us to conclude that

$$
\ell\left(S_{j}\right) \leq \frac{K+1}{2} \ell\left(S_{i}\right)
$$

But then $x \in K \frac{K+1}{2} S_{i}$, since $x \in K S_{j}$.

Remark 4.5. It is easy to see that properties (2-6) and (2-7) hold for finite unions of sets $\Omega_{i}$ (and not only for $\Omega_{i} \cup \Omega_{i+1}$ ). Therefore we may apply both properties to $\widetilde{\Omega}_{i} \cup \widetilde{\Omega}_{i+1}$, where $\widetilde{\Omega}_{i}=\widetilde{K} S_{i} \cap \Omega$. On the other hand (2-6) implies that the curve from (2-6) is contained in a finite union of sets $\Omega_{i}$ (or in a universal dilation of $S_{i}$ ).

Lemma 4.6. For each $Q \in \mathcal{W}_{3}$ there is a cube $Q^{*} \in \mathcal{W}$ such that

$$
\begin{gathered}
\frac{1}{4} \ell(Q) \leq \ell\left(Q^{*}\right) \leq \ell(Q), \\
d\left(Q^{*}, Q\right) \leq C \ell(Q) .
\end{gathered}
$$

Proof. Let $i$ be such that $z_{Q} \in\left[z_{i}, z_{i-1}\right)$, and $x \in \Omega$ such that $d(Q, x) \leq 5 \sqrt{n} \ell(Q)$. We may assume that $\varepsilon /(\sqrt{n} K)<1 / 2$. In this case, observe that

$$
x_{n} \geq z_{Q}-5 \ell(Q) \geq z_{i}-5 \frac{\varepsilon(K-1)}{5 \sqrt{n} K} \ell\left(S_{i}\right) \geq z_{i}-\frac{K-1}{2} \ell\left(S_{i}\right) .
$$

The right hand term of the equation is exactly the floor of the expanded cube $K S_{i}$. On the other hand,

$$
\begin{aligned}
x_{n} \leq z_{i-1}+5 \ell(Q) & \leq z_{i-1}+5 \frac{\varepsilon(K-1)}{5 \sqrt{n} K} \ell\left(S_{i}\right) \\
& \leq z_{i}+\ell\left(S_{i}\right)+\frac{K-1}{2} \ell\left(S_{i}\right)=z_{i}+\frac{K+1}{2} \ell\left(S_{i}\right),
\end{aligned}
$$


and the right term is the roof of the expanded cube $K S_{i}$. Consequently, $x \in \widetilde{\Omega}_{i}$. Let $y \in \widetilde{\Omega}_{i}$ be such that $|x-y|=\frac{5 \sqrt{n}}{\varepsilon} \ell(Q)$. Note that this is possible because

$$
|x-y|=\frac{5 \sqrt{n}}{\varepsilon} \ell(Q) \leq \frac{K-1}{K} \ell\left(S_{i}\right)<\operatorname{diam} \widetilde{\Omega}_{i} .
$$

Then let $\gamma$ be the curve given by properties (2-6) and (2-7). If $\xi \in \gamma$ is such that $|x-\xi|,|\xi-y| \geq|x-y| / 2$, we have $d_{\partial \Omega}(\xi) \geq \frac{\varepsilon}{4}|x-y|=(5 \sqrt{n} / 4) \ell(Q)$. If $S \in \mathcal{W}$, $S \ni \xi$, then

$$
4 \sqrt{n} \ell(S) \geq d(S, \partial \Omega) \geq d_{\partial \Omega}(\xi)-\sqrt{n} \ell(S) \geq \frac{5}{4} \sqrt{n} \ell(Q)-\sqrt{n} \ell(S) .
$$

Therefore

$$
\ell(S) \geq \frac{1}{4} \ell(Q) .
$$

Let us consider all the cubes $T \in \mathcal{W}$ satisfying $\ell(T) \geq \frac{1}{4} \ell(Q)$ and take $Q^{*}$ to be the one that minimizes the distance to $Q$. Then $\ell\left(Q^{*}\right) \leq \ell(Q)$. On the other hand

$$
d\left(Q^{*}, Q\right) \leq d(S, Q) \leq \frac{1}{\varepsilon}|x-y|+d(x, Q) \leq\left(\frac{5 \sqrt{n}}{\varepsilon^{2}}+5 \sqrt{n}\right) \ell(Q) .
$$

This completes the proof of the lemma.

Corollary 4.7. If $Q_{1}, Q_{2} \in \mathcal{W}_{3}, Q_{1} \cap Q_{2} \neq \varnothing$, then $d\left(Q_{1}^{*}, Q_{2}^{*}\right) \leq C \ell\left(Q_{1}\right)$.

The following lemma is crucial. It is analogous to Lemma 2.8 in [Jones 1981].

Lemma 4.8. Given $Q_{1}, Q_{2} \in W_{3}$ such that $Q_{1} \cap Q_{2} \neq \varnothing$, there is a constant $C=C(\varepsilon, n, K)$ and a chain of cubes $\mathscr{F}_{1,2}=\left\{V_{1}:=Q_{1}^{*}, V_{2}, \ldots, V_{r}:=Q_{2}^{*}\right\} \subset \mathcal{W}$ such that $r \leq C$ and $\ell\left(V_{i}\right) \sim \ell\left(Q_{1}\right)$ for all $i$.

Proof. Since $Q_{1} \cap Q_{2} \neq \varnothing$, we may assume that either $z_{Q_{1}}, z_{Q_{2}} \in\left[z_{i}, z_{i-1}\right)$ or that $z_{Q_{1}} \in\left[z_{i}, z_{i-1}\right)$ and $z_{Q_{2}} \in\left[z_{i+1}, z_{i}\right)$ for some $i$. In any case, thanks to Remark 4.5, $Q_{1}^{*}$ and $Q_{2}^{*}$ are not far from $\Omega_{i} \cup \Omega_{i+1}$. Let us then assume, for the sake of simplicity, that $Q_{1}^{*}, Q_{2}^{*} \subset \Omega_{i} \cup \Omega_{i+1}$. Then there is a curve $\gamma$ joining $Q_{1}^{*}$ and $Q_{2}^{*}$ with $\ell(\gamma) \leq C d\left(Q_{1}^{*}, Q_{2}^{*}\right) \leq C \ell\left(Q_{1}\right)$. Here $C$ denotes different constants, but all of them independent of the cubes considered. Let us consider the chain

$$
\mathscr{F}_{1,2}=\left\{V_{1}=Q_{1}^{*}, V_{2}, \ldots, V_{r}=Q_{2}^{*}\right\} \subset \mathscr{W}
$$

of cubes touching $\gamma$. We need a lower bound for the size of $V_{j}, j=1, \ldots, r$. We have $\ell\left(V_{2}\right) \geq \frac{1}{4} \ell\left(Q_{1}^{*}\right) \geq \frac{1}{16} \ell\left(Q_{1}\right)$. Analogously, $\ell\left(V_{r-1}\right) \geq C \ell\left(Q_{1}\right)$. If $1<j<r$, let us take $z \in \gamma \cap V_{j}$. Then

$$
d_{\partial \Omega}(z) \geq \varepsilon \frac{|x-z||z-y|}{|x-y|} \geq C \frac{\ell\left(Q_{1}\right)^{2}}{\ell\left(Q_{1}\right)} \geq C \ell\left(Q_{1}\right) .
$$

If follows that no more than $C$ cubes can be placed along $\gamma$, and then $r \leq C$. 
For each $Q_{j} \in \mathscr{W}_{3}$ let us define $P_{Q_{j}}=P\left(Q_{j}^{*}\right)$. The first term of the extension operator will be

$$
\Lambda_{1} f(x)=\sum_{Q_{j} \in W_{3}} P_{Q_{j}}(x) \xi_{j}(x) .
$$

Thanks to Lemmas 4.6 and 4.8, and Corollary 4.7, this operator can be bounded following Jones [1981] and Chua [1992]. We give the details for the sake of completeness.

Remark 4.9. In this first stage, and in particular during the proof of the next lemma, we could invoke Corollary 3.3. However, in order to be consistent with the rest of the stages we show how to use Lemma 3.2 instead.

Lemma 4.10. If $Q \in \mathcal{W}_{3}$ far from $\mathscr{W}_{4}$ (that is, $Q \in \mathcal{W}_{3}$ is surrounded by cubes in $\left.W_{3}\right)$, then

$$
\left\|D^{\alpha} \Lambda_{1} f\right\|_{L^{p}(Q)} \leq C\left\{\ell(Q)^{k-|\alpha|}\left\|\nabla^{k} f\right\|_{L^{p}(\cup \mathscr{F}(Q))}+\|f\|_{W^{k, p}\left(Q^{*}\right)}\right\},
$$

where $\mathscr{F}(Q)$ is the set of all the cubes that participate in a chain $\mathscr{F}_{j}(Q)$, connecting $Q^{*}$ with $Q_{j}^{*}$, for $Q_{j} \cap Q \neq \varnothing$.

Proof. We have

$$
\begin{aligned}
\left\|D^{\alpha} \Lambda_{1} f\right\|_{L^{p}(Q)} & =\left\|D^{\alpha} \sum_{Q_{j} \cap Q \neq \varnothing} P_{Q_{j}} \xi_{j}\right\|_{L^{p}(Q)} \\
& \leq\left\|D^{\alpha} \sum_{Q_{j} \cap Q \neq \varnothing}\left(P_{Q_{j}}-P_{Q}\right) \xi_{j}\right\|_{L^{p}(Q)}+\left\|D^{\alpha} P_{Q}\right\|_{L^{p}(Q)}=: \mathrm{I}+\mathrm{II} .
\end{aligned}
$$

The second term is easily bounded by means of Lemma 3.2, taking into account that $Q$ and $Q^{*}$ can be included inside an auxiliary cube $\tilde{Q}, Q \sim \tilde{Q} \sim Q^{*}$. Alternating the derivatives of $f$ we get

$$
\begin{aligned}
\mathrm{II} & \leq C \sum_{|\gamma+\alpha|<k} \ell(Q)^{|\gamma|}\left\|D^{\gamma+\alpha} P_{Q}\right\|_{L^{p}\left(Q^{*}\right)} \\
& \leq C \sum_{|\gamma+\alpha|<k} \ell(Q)^{|\gamma|}\left\{\left\|D^{\gamma+\alpha}\left(P_{Q}-f\right)\right\|_{L^{p}\left(Q^{*}\right)}+\left\|D^{\gamma+\alpha} f\right\|_{L^{p}\left(Q^{*}\right)}\right\} \\
& \leq C\left\|\nabla^{k} f\right\|_{L^{p}\left(Q^{*}\right)} \ell(Q)^{k-|\alpha|}+\|f\|_{W^{k, p}\left(Q^{*}\right)} \leq C\|f\|_{W^{k, p}\left(Q^{*}\right)} .
\end{aligned}
$$

On the other hand,

$$
\begin{aligned}
\mathrm{I} & \leq C \sum_{Q_{j} \cap Q \neq \varnothing} \sum_{\beta \leq \alpha}\left\|D^{\alpha-\beta} \xi_{j} D^{\beta}\left(P_{Q_{j}}-P_{Q}\right)\right\|_{L^{p}(Q)} \\
& \leq C \sum_{Q_{j} \cap Q \neq \varnothing} \sum_{\beta \leq \alpha} \frac{1}{\ell(Q)^{|\alpha-\beta|}}\left\|D^{\beta}\left(P_{Q_{j}}-P_{Q}\right)\right\|_{L^{p}(Q)} .
\end{aligned}
$$


For each $j$, let us alternate the polynomials associated to the cubes of the chain between $Q_{j}^{*}$ and $Q^{*}$ given by Lemma 4.8. We set $\mathscr{F}_{j}=\left\{T_{1}=Q^{*}, T_{2}, \ldots, T_{r}=Q_{j}^{*}\right\}$ and obtain

$$
\begin{aligned}
& \left\|D^{\beta}\left(P_{Q_{j}}-P_{Q}\right)\right\|_{L^{p}(Q)} \\
& \leq \sum_{i=1}^{r-1}\left\|D^{\beta}\left(P\left(T_{i+1}\right)-P\left(T_{i}\right)\right)\right\|_{L^{p}(Q)} \\
& \leq \sum_{i=1}^{r-1}\left\{\left\|D^{\beta}\left(P\left(T_{i+1}\right)-P\left(T_{i} \cup T_{i+1}\right)\right)\right\|_{L^{p}(Q)}+\| D^{\beta}\left(\left(P\left(T_{i} \cup T_{i+1}\right)-P\left(T_{i}\right)\right) \|_{L^{p}(Q)}\right\}\right. \\
& \leq C \sum_{i=1}^{r-1}\left\{\left\|D^{\beta}\left(P\left(T_{i+1}\right)-P\left(T_{i} \cup T_{i+1}\right)\right)\right\|_{L^{p}\left(T_{i+1}\right)}+\| D^{\beta}\left(\left(P\left(T_{i} \cup T_{i+1}\right)-P\left(T_{i}\right) \|_{L^{p}\left(T_{i}\right)}\right\}\right.\right. \\
& \leq C \sum_{i=1}^{r-1}\left\{\left\|D^{\beta}\left(P\left(T_{i+1}\right)-f\right)\right\|_{L^{p}\left(T_{i+1}\right)}+\left\|D^{\beta}\left(f-P\left(T_{i} \cup T_{i+1}\right)\right)\right\|_{L^{p}\left(T_{i} \cup T_{i+1}\right)} \quad+\left\|D^{\beta}\left(f-P\left(T_{i}\right)\right)\right\|_{L^{p}\left(T_{i}\right)}\right\} \\
& \leq C \sum_{i=1}^{r-1} \ell(Q)^{k-|\beta|}\left\|\nabla^{k} f\right\|_{L^{p}\left(T_{i} \cup T_{i+1}\right)} \leq \ell(Q)^{k-|\beta|}\left\|\nabla^{k} f\right\|_{L^{p}\left(\cup \mathscr{F}_{j}\right) .}
\end{aligned}
$$

And then

$$
\mathrm{I} \leq C \ell(Q)^{k-|\alpha|}\left\|\nabla^{k} f\right\|_{L^{p}(\mathrm{UF}(Q))} .
$$

Finally, let us observe that from Lemmas 4.6 and 4.8 it follows that

$$
\begin{aligned}
& \left\|\sum_{\substack{Q_{l} \in \mathscr{W}_{3} \\
Q_{l} \cap Q_{j} \neq \varnothing}} \chi \cup \mathscr{F}_{j l}\right\|_{\infty} \leq C<\infty \quad \text { for all } Q_{j} \in \mathscr{W}_{3}, \\
& \left\|\sum_{Q_{j} \in \mathscr{W}_{3}} \chi \cup \mathscr{F}\left(Q_{j}\right)\right\|_{\infty} \leq C<\infty .
\end{aligned}
$$

This means that each cube $Q_{j}^{*}$ is used at most a fixed number of times. Then

therefore

$$
\left\|D^{\alpha} \Lambda_{1} f\right\|_{L^{p}\left(\cup^{\left.W_{3}\right)}\right.}^{p}=\sum_{Q \in W_{3}}\left\|D^{\alpha} \Lambda_{1} f\right\|_{L^{p}(Q)}^{p} \leq C\|f\|_{W^{k, p}(\Omega)}^{p},
$$

$$
\left\|D^{\alpha} \Lambda_{1} f\right\|_{L^{p}\left(\cup W_{3}\right)} \leq C\|f\|_{W^{k, p}(\Omega)} .
$$

Hence, the operator $\left(\Lambda_{1}\right)$ is bounded far from $\mathcal{W}_{4}$.

Second stage. This stage, where the extension operator is defined over $\mathcal{W}_{4}$, is the heart of the extension process. The first stage was essentially a translation of Jones's theorem, which extends functions to an expanded cusp, where no weight is needed. The second stage, on the other hand, extends functions to a cone: here 
the cuspidal behavior of $\Omega$ is compensated by a weight. The third stage, in turn, completes the extension to a neighborhood of the origin, but does not contain any interesting ideas: we detail it for the sake of completeness.

Let us begin stating some properties of $\mathscr{W}_{4}$ itself. Let $T$ be a cube in $\mathscr{W}_{4}$, and let $S_{i}=S\left(z_{T}\right)$. Observe that from the definition of $\mathcal{W}_{3}$ we know that $\ell(T)>C \ell\left(S_{i}\right)$, with the constant $C=\frac{\varepsilon}{5 \sqrt{n}} \frac{K-1}{K}$. In order to simplify notation in subsequent calculations we set $C=1$ and assume that $\ell(T) \geq \ell\left(S_{i}\right)$.

Let $\widetilde{W}_{4}$ denote de Whitney decomposition of $\mathbb{R}^{n} \backslash \hat{x}_{n}$. Observe that the structure of $\widetilde{W}_{4}$ is very simple: cubes grow exponentially as we move away from the axis. Since the positive semiaxis of $\hat{x}_{n}$ is contained in $\Omega$, Remark 2.4 implies that for every cube $T \in \mathscr{W}_{4}$, there is a cube $\widetilde{T} \in \widetilde{W}_{4}$ such that $T \subset \widetilde{T}$. The following lemma proves that in fact $\ell(T) \sim \ell(\widetilde{T})$ for all $T \in \mathscr{W}_{4}$.

Lemma 4.11. There is a constant $C$ such that $d\left(T, \hat{x}_{n}\right) \leq C \ell(T)$ for all $T$ in $W_{4}$.

Proof. Let $x^{*} \in \partial \Omega$ be such that $d(T, \partial \Omega)=d\left(T, x^{*}\right)$. Let $\gamma$ be the segment joining $T$ and $x^{*}$, and $Q \in \mathcal{W}_{3}$, the nearest cube to $T$ such that $Q \cap \gamma \neq \varnothing$. It is clear that $\ell(Q) \leq d(Q, \partial \Omega) \leq d(T, \partial \Omega) \leq 4 \sqrt{n} \ell(T)$. Let us denote by $x^{q} \in \partial \Omega$ the point such that $d(Q, \partial \Omega)=d\left(Q, x^{q}\right)$. Then

$$
\begin{aligned}
d\left(T, \hat{x}_{n}\right) & \leq d(T, Q)+\sqrt{n} \ell(Q)+d\left(Q, \hat{x}_{n}\right) \\
& \leq 4 \sqrt{n} \ell(T)+4 n \ell(T)+d\left(Q, x^{q}\right)+d\left(x^{q}, \hat{x}_{n}\right) \\
& \leq C \ell(T)+d\left(x^{q}, \hat{x}_{n}\right) \leq C \ell(T)+\widetilde{K} \ell\left(S\left(z_{Q}\right)\right) .
\end{aligned}
$$

Consequently, if $\ell\left(S\left(z_{Q}\right)\right) \leq C \ell(T)$, the result is proved.

Let us set $I=i_{z_{Q}}$. Furthermore, let $T_{1} \in \mathcal{W}_{4}$ be such that $T_{1} \cap Q \neq \varnothing$ and $T_{1} \cap \gamma \neq \varnothing$. Then $\frac{1}{4} \ell(Q) \leq \ell\left(T_{1}\right) \leq 4 \ell(Q)$. Suppose that $\ell(Q)<\frac{1}{16} \ell\left(S_{I}\right)$. Then

$$
z_{T_{1}} \geq z_{Q}-\ell\left(T_{1}\right) \geq z_{I}-4 \ell(Q)>z_{I}-\frac{1}{4} \ell\left(S_{I}\right) \geq z_{I}-\ell\left(S_{I+1}\right) \geq z_{I+1} .
$$

But, since $T_{1} \in \mathscr{W}_{4}$,

$$
\ell(Q) \geq \frac{1}{4} \ell\left(T_{1}\right) \geq \frac{1}{4} \ell\left(S\left(z_{T_{1}}\right)\right) \geq \frac{1}{4} \ell\left(S_{I+1}\right) \geq \frac{1}{16} \ell\left(S_{I}\right),
$$

which is a contradiction. Consequently, $\ell(T) \geq C \ell(Q) \geq C \ell\left(S_{I}\right)$, and the result follows.

Remark 4.12. A much simpler proof for this lemma can be provided assuming property (2-13). However, item (i) in Theorem 4.1 can be proved without (2-13), and so we prefer to detail the general proof.

As we stated above, Lemma 4.11 shows that $\ell(T) \sim \ell(\widetilde{T})$ for all $T \in \mathcal{W}_{4}$. This fact implies that the number of cubes of a certain size in $\mathscr{W}_{4}$ is comparable with the number of cubes of the same size in $\widetilde{W}_{4}$. In some passages of this stage, we 
estimate the number of cubes in $\mathscr{W}_{4}$ by the number of cubes in $\widetilde{W}_{4}$, which are easier to count.

In this second stage a weight is needed in order to bound the norm of the extension operator: we provide two different versions of the extension to the cubes in $\mathscr{W}_{4}$, the first one is horizontal (each cube will be associated with a set at the same height), leading to the weight $\sigma(x)=(\ell(S(|x|)) /|x|)^{(n-1) / p}$, corresponding to Theorem 4.1(b). Property (2-13) is needed in this case. The second version is vertical, giving another possible weight $\sigma(x)=(\ell(S(|x|)) /|x|)^{k}$, as in Theorem 4.1(a). Property (2-13) is not needed for this version.

First version: dimensional-horizontal weight. For each cube $T_{j} \in \mathscr{W}_{4}$ let us define

$$
S\left(T_{j}\right)=\bigcup\left\{S_{i}: z_{T_{j}} \leq z_{i}<z_{T_{j}}+\ell\left(T_{j}\right)\right\} .
$$

Remark 4.13. $S\left(T_{j}\right)$ is the reflected set of $T_{j}$ and $Q_{j}^{*}$ is the reflected cube of $Q_{j}$ in the first stage. Observe that $S\left(T_{j}\right)$ is not a cube, nor a rectangle. However, the normality property (2-3) implies that it is a tower of cubes, eventually of different sizes. Since cubes in $\mathcal{W}_{4}$ are far from $\Omega, T_{j}$ will be larger than the $S_{i}$ 's in $S\left(T_{j}\right)$. Nevertheless, the dyadic nature of cubes in Whitney decompositions implies that its height is exactly $\ell\left(T_{j}\right)$. Finally, if $S\left(T_{j}\right)=\left\{S_{I_{j}}, S_{I_{j}+1} \ldots, S_{I_{j}+N_{j}}\right\}$, then property (2-13) guarantees that $\ell\left(S_{I_{j}}\right) / \ell\left(S_{I_{j}+N_{j}}\right) \leq C<\infty$. Therefore, for each $T_{j}$ there is a pair of rectangles $R_{j}^{1}$ and $R_{j}^{2}$ such that

$$
\begin{aligned}
R_{j}^{1} & \subset S\left(T_{j}\right) \subset R_{j}^{2}, \\
\vec{\ell}\left(R_{j}^{1}\right) & =\left(\ell\left(S_{I_{j}+N_{j}}\right), \ldots, \ell\left(S_{I_{j}+N_{j}}\right), \ell\left(T_{j}\right)\right), \\
\vec{\ell}\left(R_{j}^{2}\right) & =\left(\ell\left(S_{I_{j}}\right), \ldots, \ell\left(S_{I_{j}}\right), \ell\left(T_{j}\right)\right),
\end{aligned}
$$

satisfying $\ell_{i}\left(R_{j}^{2}\right) / \ell_{i}\left(R_{j}^{1}\right) \leq C$ for all $T_{j}$ and $i=1, \ldots, n$; in other words, $R_{j}^{1} \sim R_{j}^{2}$.

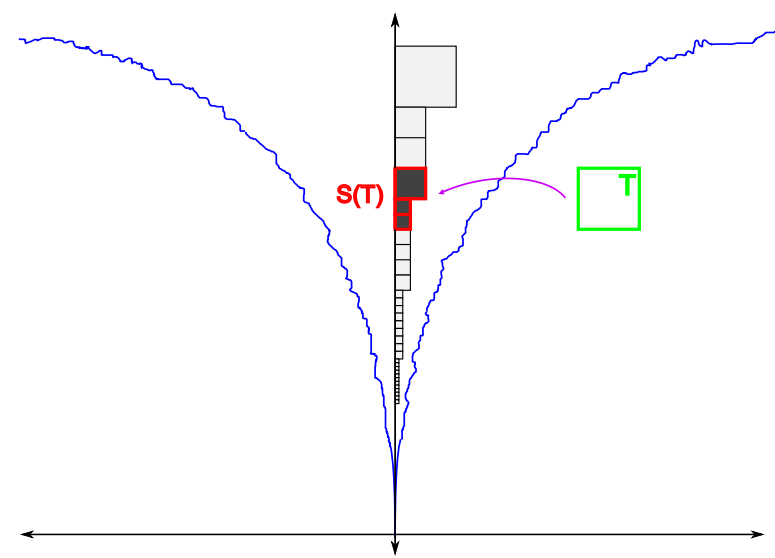

Figure 2. Reflected tower: The second stage's first version. 
Let us define, for each $T_{j} \in \mathcal{W}_{4}, P_{T_{j}}=P\left(R_{j}^{1}\right)$. Our extension operator is

for $x \in T \in \mathscr{W}_{4}$.

$$
\Lambda_{2} f(x)=\sum_{T_{j} \in \mathscr{W}_{4}} P_{T_{j}}(x) \varphi_{j}(x)
$$

The following lemma is equivalent to Lemma 4.10. However, since $\mathcal{W}_{4}$ is far from $\Omega$, a weight is needed.

Lemma 4.14. If $T \in \mathcal{W}_{4}\left(\right.$ far from $\mathcal{W}_{3}$ and $\left.\mathcal{W}_{5}\right)$, then

$$
\left\|D^{\alpha} \Lambda_{2} f\right\|_{L^{p}(T)} \leq C\left(\frac{|T|}{\left|R^{1}\right|}\right)^{1 / p}\|f\|_{W^{k, p}(\cup \mathscr{F}(T))},
$$

where $\mathscr{F}(T)$ is the union of all the $S\left(T_{j}\right)$ with $T_{j} \cap T \neq \varnothing$, and $R^{1}$ is the rectangle in $S(T)$ provided by Remark 4.13.

Proof. As we proceeded in Lemma 4.10, we alternate the polynomial $P_{T}$ corresponding to $T$. We have

$$
\begin{aligned}
\left\|D^{\alpha} \Lambda_{2} f\right\|_{L^{p}(T)} & =\left\|D^{\alpha} \sum_{T_{j} \cap T \neq \varnothing} P_{T_{j}} \varphi_{j}\right\|_{L^{p}(T)} \\
& \leq\left\|D_{T_{j} \cap T \neq \varnothing}^{\alpha}\left(P_{T_{j}}-P_{T}\right) \varphi_{j}\right\|_{L^{p}(T)}+\left\|D^{\alpha} P_{T}\right\|_{L^{p}(T)}=: \mathrm{I}+\mathrm{II} .
\end{aligned}
$$

Since $d(T, S(T)) \leq C \ell(T)$, the second term can be bounded by means of Lemma 3.2 , by considering an auxiliary cube $\tilde{T} \sim T$ such that $T, S(T) \subset \tilde{T}$. We get

$$
\mathrm{II} \leq C\left(\frac{|T|}{\left|R^{1}\right|}\right)^{1 / p} \sum_{\gamma:|\gamma+\alpha|<k} \ell(T)^{|\gamma|}\left\|D^{\alpha+\gamma} P_{T}\right\|_{L^{p}(S(T))} .
$$

If we go on as in Lemma 4.10, then we obtain

$$
\mathrm{II} \leq C\left(\frac{|T|}{\left|R^{1}\right|}\right)^{1 / p}\|f\|_{W^{k, p}(S(T))} .
$$

On the other hand,

$$
\mathrm{I} \leq C \sum_{T_{j} \cap T \neq \varnothing} \sum_{\beta \leq \alpha} \frac{1}{\ell(T)^{|\alpha-\beta|}}\left\|D^{\beta}\left(P_{T_{j}}-P_{T}\right)\right\|_{L^{p}(T)},
$$

and $T \cap T_{j} \neq \varnothing$, implies that $S(T) \cap S\left(T_{j}\right) \neq \varnothing$ and $R^{1} \sim R_{j}^{1}$. In fact, $S(T) \subset S\left(T_{j}\right)$ or $S\left(T_{j}\right) \subset S(T)$ (which imply $R^{1} \subset R_{j}^{1}$ or $R_{j}^{1} \subset R^{1}$ respectively), or $S(T)$ and $S\left(T_{j}\right)$ form a new, longer tower where $S(T)$ is over $S\left(T_{j}\right)$, or vice versa (which implies that $R^{1}$ and $R_{j}^{1}$ are touching). We only treat the case that leads to touching 
rectangles (the other cases follow similarly):

$$
\begin{aligned}
& \left\|D^{\beta}\left(P_{T_{j}}-P_{T}\right)\right\|_{L^{p}(T)} \\
& \leq\left\|D^{\beta}\left(P_{T_{j}}-P\left(R^{1} \cup R_{j}^{1}\right)\right)\right\|_{L^{p}(T)}+\left\|D^{\beta}\left(P_{T}-P\left(R^{1} \cup R_{j}^{1}\right)\right)\right\|_{L^{p}(T)} \\
& \leq C\left(\frac{|T|}{\left|R^{1}\right|}\right)^{1 / p} \sum_{\gamma:|\gamma+\beta|<k} \ell(T)^{|\gamma|}\left\{\left\|D^{\beta+\gamma}\left(P_{T}-P\left(R^{1} \cup R_{j}^{1}\right)\right)\right\|_{L^{p}\left(R^{1}\right)}\right. \\
& \left.+\left\|D^{\beta+\gamma}\left(P_{T_{j}}-P\left(R^{1} \cup R_{j}^{1}\right)\right)\right\|_{L^{p}\left(R_{j}^{1}\right)}\right\} \\
& \leq C\left(\frac{|T|}{\left|R^{1}\right|}\right)^{1 / p} \sum_{\gamma:|\gamma+\beta|<k} \ell(T)^{|\gamma|}\left\{\left\|D^{\beta+\gamma}\left(P_{T}-f\right)\right\|_{L^{p}\left(R^{1}\right)}\right. \\
& +\left\|D^{\gamma+\beta}\left(f-P\left(R^{1} \cup R_{j}^{1}\right)\right)\right\|_{L^{p}\left(R^{1}\right)} \\
& +\left\|D^{\beta+\gamma}\left(P_{T_{j}}-f\right)\right\|_{L^{p}\left(R_{j}^{1}\right)} \\
& \left.+\left\|D^{\gamma+\beta}\left(f-P\left(R^{1} \cup R_{j}^{1}\right)\right)\right\|_{L^{p}\left(R_{j}^{1}\right)}\right\} .
\end{aligned}
$$

Applying Lemma 3.5 we obtain

$\left\|D^{\beta}\left(P_{T_{j}}-P_{T}\right)\right\|_{L^{p}(T)}$

$$
\leq C\left(\frac{|T|}{\left|R^{1}\right|}\right)^{1 / p} \sum_{\gamma:|\gamma+\beta|<k} \ell(T)^{|\gamma|} \ell\left(R^{1}\right)^{k-|\gamma|-|\beta|} \sum_{\tau:|\tau|=k}\left\|D^{\tau} f\right\|_{L^{p}\left(S(T) \cup S\left(T_{j}\right)\right)} .
$$

Consequently

$$
\begin{aligned}
\mathrm{I} & \leq C \sum_{T_{j} \cap T \neq \varnothing|\beta| \leq|\alpha|} \sum_{\gamma:|\gamma+\beta| \leq k} \frac{1}{\ell(T)^{|\alpha-\beta|}} C\left(\frac{|T|}{\left|R^{1}\right|}\right)^{1 / p} \\
& \sum_{T_{j} \cap T \neq \varnothing|\beta| \leq|\alpha|} \ell(T)^{|\gamma|} \ell\left(R^{1}\right)^{k-|\gamma|-|\beta|} \sum_{\tau:|\tau|=k}\left\|D^{\tau} f\right\|_{L^{p}\left(S(T) \cup S\left(T_{j}\right)\right)} \\
& \leq C \sum_{\gamma\left(\frac{|T|}{\left|R^{1}\right|}\right)^{1 / p}\|f\|_{W^{k, p}(\mathscr{F}(T))}} \sum^{|k|-|\alpha|} C\left(\frac{|T|}{\left|R^{1}\right|}\right)^{1 / p} \sum_{\gamma:|\gamma+\beta|<k} \sum_{\tau:|\tau|=k}\left\|D^{\tau} f\right\|_{L^{p}\left(S(T) \cup S\left(T_{j}\right)\right)}
\end{aligned}
$$

Lemma 4.10 bounds the norm of the extension operator in all the cubes in $\mathscr{W}_{3}$ far from $\mathscr{W}_{4}$, that is, in all cubes $Q \in W_{3}$ such that all the neighbors of $Q$ are in $\mathscr{W}_{3}$. Lemma 4.14 does the same thing for cubes in $\mathscr{W}_{4}$, far from $\mathscr{W}_{3}$. Let us consider now cubes in the frontier of these sets: let $Q \in \mathcal{W}_{3}$ and $T \in \mathcal{W}_{4}$ be such that $Q \cap T \neq \varnothing$. Notice that $\frac{1}{4} \leq \ell(Q) / \ell(T) \leq 4$. Furthermore,

$$
4 \sqrt{n} \ell(Q) \geq d(Q, \partial \Omega) \geq d(T, \partial \Omega)-\sqrt{n} \ell(Q) \geq \ell(T)-\sqrt{n} \ell(Q),
$$

and then

$$
\ell(T) \leq 5 \sqrt{n} \ell(Q) \leq C \ell\left(S_{I}\right)
$$


where $S_{I}$ is the cube in the spine of $\Omega$ such that $z_{Q} \in\left[z_{I}, z_{I-1}\right)$. This implies that $\ell\left(Q^{*}\right) \sim \ell(T)$, and since $S_{I} \cap S(T) \neq \varnothing$, by means of Lemma 4.8, there is a chain of cubes joining $Q^{*}$ and $S(T)$. Hence, the proof of the following lemma is the same that the one of Lemmas 4.10 and 4.14.

Lemma 4.15. Let $Q \in \mathscr{W}_{3}$ and $T \in \mathscr{W}_{4}$ be such that $Q \cap T \neq \varnothing$, then

$$
\begin{aligned}
\left\|D^{\alpha}\left(\Lambda_{1}+\Lambda_{2}\right) f\right\|_{L^{p}(Q)} & \leq C\|f\|_{W^{k, p}(\mathscr{F}(Q))}, \\
\left\|D^{\alpha}\left(\Lambda_{1}+\Lambda_{2}\right) f\right\|_{L^{p}(T)} & \leq C\|f\|_{W^{k, p}(\mathscr{F}(T))} .
\end{aligned}
$$

We need to prove that the norm of the extension is bounded as in Lemma 4.14 all over $\mathscr{W}_{4}$ and not only in a particular cube. Let us pick a cube $S_{i} \in \mathscr{Y}$. A simple comparison with $\widetilde{W}_{4}$ implies that the number of cubes $T_{j}$ with $\ell\left(T_{j}\right)=2^{m} \ell\left(S_{i}\right)$, such that $S_{i} \subset S\left(T_{j}\right)$, is bounded by a constant independent of $S_{i}$. Furthermore, such a comparison allows us to bound the possible values of $m$, for each $i$, by $0 \leq m \leq \log \left(z_{i} / \ell\left(S_{i}\right)\right)$, where $\log =\log _{2}$.

Proposition 4.16. If we set $\sigma(x)=(\ell(S(|x|)) /|x|)^{(n-1) / p}$, then

$$
\left\|\sigma D^{\alpha} f\right\|_{L^{p}\left(\cup W_{4}\right)} \leq C\|f\|_{W^{k, p}(\cup \varphi)} .
$$

Proof. We can take $\sigma$ as constant in each cube $T \in \mathcal{W}_{4}: \sigma_{T} \sim\left(\frac{\ell\left(S\left(z_{T}\right)\right)}{z_{T}}\right)^{(n-1) / p}$. Then

$$
\begin{aligned}
\left\|\sigma D^{\alpha} f\right\|_{L^{p}\left(\cup W_{4}\right)}^{p}=\sum_{T \in \mathscr{W}_{4}}\left\|\sigma D^{\alpha} f\right\|_{L^{p}(T)}^{p} & \leq C \sum_{T \in W_{4}}\left(\frac{\ell\left(S\left(z_{T}\right)\right)}{z_{T}}\right)^{n-1}\left\|D^{\alpha} f\right\|_{L^{p}(T)}^{p} \\
& \leq C \sum_{T \in W_{4}}\left(\frac{\ell\left(S\left(z_{T}\right)\right)}{z_{T}}\right)^{n-1} \frac{|T|}{\left|R^{1}\right|}\|f\|_{W^{k, p}(\cup \mathscr{F}(T))}^{p} .
\end{aligned}
$$

Now, since $\ell_{i}\left(R^{1}\right) \sim \ell\left(S\left(z_{T}\right)\right)$ for $i=1, \ldots, n-1$, and $\ell_{n}\left(R^{1}\right)=\ell(T)$, we have

$$
\begin{aligned}
\left\|\sigma D^{\alpha} f\right\|_{L^{p}\left(\cup^{\left.W_{4}\right)}\right.}^{p} & \leq C \sum_{T \in \mathscr{W}_{4}}\left(\frac{\ell(T)}{z_{T}}\right)^{n-1}\|f\|_{W^{k, p}(\cup \mathscr{F}(T))}^{p} \\
& =C \sum_{T \in \mathscr{W}_{4}} \sum_{S \in \mathscr{F}(T)}\left(\frac{\ell(T)}{z_{T}}\right)^{n-1}\|f\|_{W^{k, p}(S)}^{p} \\
& =C \sum_{S \in \mathscr{Y}} \sum_{T: \mathscr{F}(T) \ni S}\left(\frac{\ell(T)}{z_{T}}\right)^{n-1}\|f\|_{W^{k, p}(S)}^{p} .
\end{aligned}
$$

Given a fixed cube $S \in \mathscr{Y}$, the cubes $T$ in $\mathscr{W}_{4}$ can be classified by their sizes $\ell(T)=2^{m} \ell(S)$, where $0 \leq m \leq M=\log \left(z_{S} / \ell(S)\right)$. Furthermore, $z_{T} \sim z_{S}$ for every $T \in \mathscr{W}_{4}$ such that $S \in \mathscr{F}(T)$. Finally, the comparison between cubes in $\mathscr{W}_{4}$ and cubes in $\widetilde{W}_{4}$ guarantees that, given a cube $S \in \mathscr{Y}$, there is a bound $C$, depending 
only on the dimension $n$, such that

$$
\#\left\{T \in \mathscr{W}_{4}: S \in \mathscr{F}(T), \ell(T)=2^{m} \ell(S)\right\} \leq C .
$$

Then

$$
\begin{aligned}
\left\|\sigma D^{\alpha} f\right\|_{L^{p}\left(\cup^{w} W_{4}\right)}^{p} & \leq C \sum_{S \in \mathscr{S}} \sum_{m=1}^{M-1} \sum_{\substack{T: \mathscr{F}(T) \ni S \\
\ell(T)=2^{m} \ell(S)}}\left(\frac{\ell(T)}{z_{T}}\right)^{n-1}\|f\|_{W^{k, p}(S)}^{p} \\
& \leq C \sum_{S \in \mathscr{S}} z_{S}^{1-n} \ell(S)^{n-1}\left(\sum_{m=1}^{M-1}\left(2^{n-1}\right)^{m}\right)\|f\|_{W^{k, p}(S)}^{p},
\end{aligned}
$$

and the result follows by recalling that $M=\log \left(z_{S} / \ell(S)\right)$.

This result concludes the first version of the second stage of the extension.

Second version: derivative-vertical weight. This version of the extension is based on a different construction of the reflected set of a cube in $W_{4}$. For each $T$, we find some $T^{*} \in \mathscr{Y}$ such that $\ell\left(T^{*}\right) \sim \ell(T)$, but $T^{*}$ is far above $T$. The weight in this case is due to the distance between $T$ and $T^{*}$.

Let us consider $\tilde{T}$ a cube belonging to $\widetilde{W}_{4}$ (the Whitney decomposition of $\mathbb{R}^{n} \backslash \hat{x}_{n}$ ) such that $\tilde{T} \cap T \neq \varnothing$ for some $T \in \mathcal{W}_{4}$. Thanks to Lemma 4.11 only a finite number (the number does not depend on $\tilde{T}$ ) of cubes that belong to $\mathscr{W}_{4}$ are contained in $\tilde{T}$. We can now pack the elements of $\mathscr{W}_{4}$ into cylinders of the form $\eta(\tilde{T})=Q^{\prime} \times \mathbb{R}$, where $Q^{\prime} \subset \mathbb{R}^{n-1}$ is the projected face $F_{\tilde{T}}^{u}$ of $\tilde{T}$ onto $\mathbb{R}^{n-1}$. We identify cylinders given by cubes $\tilde{T}$ that share a projection $Q^{\prime}$. In this way each cube $T \in W_{4}$ belongs to only one cylinder. Moreover, cubes inside the cylinder $\eta(\tilde{T})$ are equivalent, that is, $T_{1}, T_{2} \in \eta(\tilde{T})$ implies that $T_{1} \sim \tilde{T} \sim T_{2}$. For each

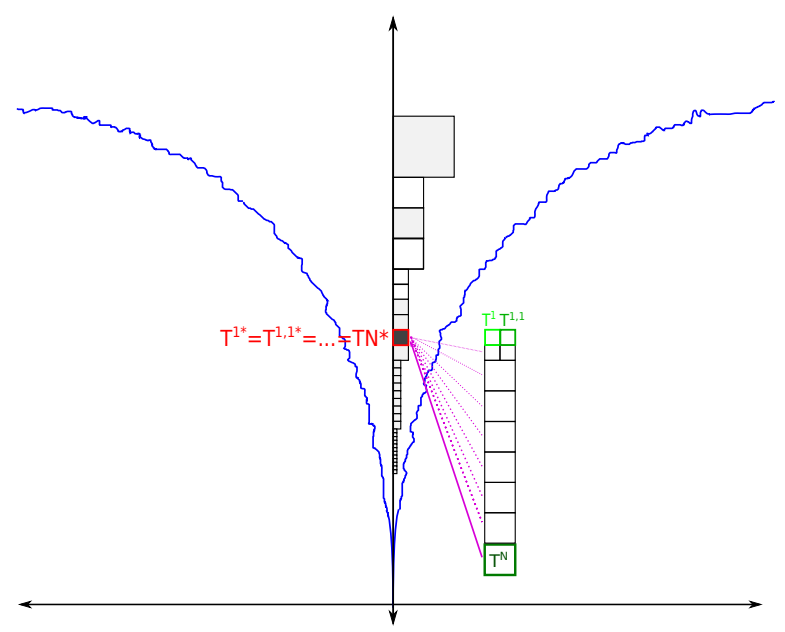

Figure 3. Reflected cubes: The second stage's second version. 
$T_{j} \in \mathcal{W}_{4}$, we denote by $\tau\left(T_{j}\right)$ the set of cubes in $\mathscr{W}_{4}$ that share a cylinder with $T_{j}$. The set $\tau$ is called a tower.

Let us consider one of the upper cubes $T^{1}$ in $\tau\left(T^{1}\right)$. We define $T^{*}=S\left(z_{T^{1}}\right)$ for every $T \in \tau\left(T^{1}\right)$. This situation is represented in Figure 3 .

It is important that, with this definition, for every $T \in \mathcal{W}_{4}$ we have $T^{*} \in \mathscr{Y}$, and $T^{*} \sim T$. However, the distance between $T$ and $T^{*}$ could be large, particularly in the $x_{n}$ direction. In fact, since $d\left(T^{1}, T^{N}\right) \sim z_{T^{1}}$, we have $d\left(T^{1}, T^{1 *}\right) \sim \ell\left(T^{1}\right)$, but $d\left(T^{N}, T^{N *}\right) \sim z_{T^{1}}$ (where $T^{1}$ and $T^{N}$ are upper and lower cubes in a certain tower $\tau$ ).

As in the first version of the second stage extension, we define $P_{T_{j}}=P\left(T_{j}^{*}\right)$ and

$$
\Lambda_{2} f(x)=\sum_{T_{j} \in \mathscr{W}_{4}} P_{T_{j}}(x) \varphi_{j}(x) .
$$

Observe that if $T_{j}, T \in \mathcal{W}_{4}$ and $T_{j} \cap T \neq \varnothing$, the tops of the towers $\tau(T)$ and $\tau\left(T_{j}\right)$ could be settled at very different heights (and so could the heights of the reflected cubes $T_{j}^{*}$ and $T^{*}$ ). This is because of the following fact.

Remark 4.17. Suppose $S \in \mathscr{Y}$ is the higher cube of a certain size. Let us denote by $c(S)$ the number of cubes with edges of length exactly $\ell(S)$. Then, since $0 \in \partial \Omega$, $z_{S}-c(S) \ell(S)>0$, and consequently $c(S) \leq z_{S} / \ell(S)$. However, no better estimate can be provided (in fact, it is easy to see that for cusps with profile $\varphi(z)=z^{\nu}$, there are $\sim z^{1-v}$ cubes with edges $z^{v}$ ), so the worst case, that there could be $\sim z_{S} / \ell(S)$ cubes in $\mathscr{S}$ with side $\ell(S)$, should be assumed to hold.

Consequently, the shape of $\mathcal{W}_{4}$ could show long steps. When two towers touching each other are in the edge of a long step, their heights are very different. This situation is represented in Figure 4. In this figure, two touching towers are shown,

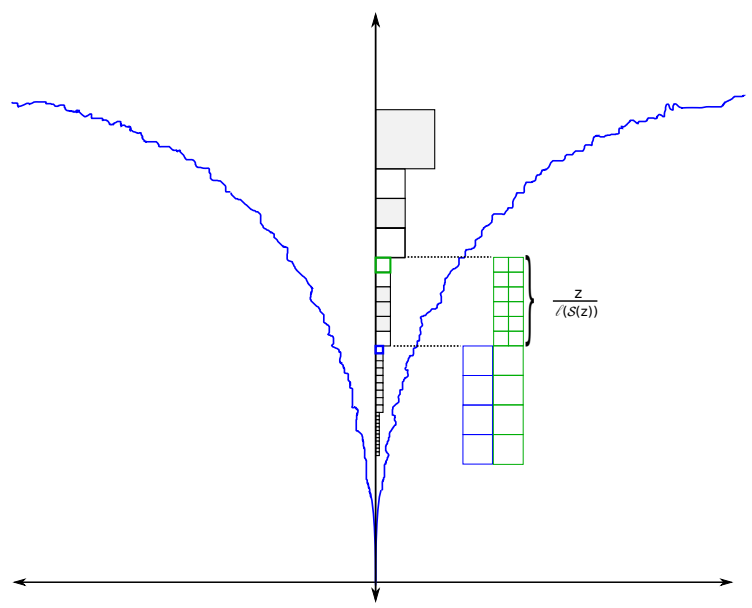

Figure 4. Long steps imply long chains. 
where reflected cubes are far from each other. Therefore, the chain (in $\mathscr{S}$ ) joining the reflected cubes for each tower is large.

Lemma 4.18. For every cube $T \in \mathcal{W}_{4}$,

$$
\left\|D^{\alpha} \Lambda_{2} f\right\|_{L^{p}(T)} \leq C \ell(T)^{k-|\alpha|}\left(\frac{\ell_{n}(\tau(T))}{\ell(T)}\right)^{k-1 / p}\|f\|_{W^{k, p}(\cup \mathscr{F}(T))},
$$

where $\mathscr{F}(T)$ is the union of the cubes in all the chains connecting $T^{*}$ and $T_{j}^{*}$ for $T_{j} \cap T \neq \varnothing$.

Proof. We have

$$
\left\|D^{\alpha} \Lambda_{2} f\right\|_{L^{p}(T)} \leq\left\|D^{\alpha} \sum_{T_{j} \cap T \neq \varnothing}\left(P_{T_{j}}-P_{T}\right) \varphi_{j}\right\|_{L^{p}(T)}+\left\|D^{\alpha} P_{T}\right\|_{L^{p}(T)}=: \mathrm{I}+\mathrm{II} .
$$

As usual,

$$
\mathrm{I} \leq C \sum_{\beta \leq \alpha} \frac{1}{\ell(T)^{|\alpha|-|\beta|}} \sum_{T_{j} \cap T \neq \varnothing}\left\|D^{\beta}\left(P_{T_{j}}-P_{T}\right)\right\|_{L^{p}(T)} .
$$

Let us denote by $\mathscr{F}_{j}(T)=\left\{T^{*}=S^{1}, S^{2}, \ldots, T_{j}^{*}=S^{M}\right\}$ the chain of cubes joining $T^{*}$ and $T_{j}^{*}$, then

$$
\left\|D^{\beta}\left(P_{T_{j}}-P_{T}\right)\right\|_{L^{p}(T)} \leq \sum_{l=1}^{M-1}\left\|D^{\beta}\left(P\left(S^{l+1}\right)-P\left(S^{l}\right)\right)\right\|_{L^{p}(T)} .
$$

Now, if we denote by $R_{l}$ the minimal rectangle containing $T$ and $S^{l}$, we have $\ell_{i}\left(R_{l}\right) \sim \ell(T), i=1, \ldots, n-1$, and $\ell_{n}(R) \leq \ell_{n}(\tau(T))$. Then

$$
\begin{aligned}
& \left\|D^{\beta}\left(P\left(S^{l+1}\right)-P\left(S^{l}\right)\right)\right\|_{L^{p}(T)} \\
& \left.\leq C\left\{\left\|D^{\beta}\left(P\left(S^{l+1}\right)-P\left(S^{l+1} \cup S^{l}\right)\right)\right\|_{L^{p}(T)}+\| P\left(S^{l+1} \cup S^{l}\right)-P\left(S^{l}\right)\right) \|_{L^{p}(T)}\right\} \\
& \leq C|T|^{\frac{1}{p}}\left\{\left\|D^{\beta}\left(P\left(S^{l+1}\right)-P\left(S^{l+1} \cup S^{l}\right)\right)\right\|_{L^{\infty}\left(R_{l+1}\right)}\right. \\
& \left.+\left\|D^{\beta}\left(P\left(S^{l+1} \cup S^{l}\right)-P\left(S^{l}\right)\right)\right\|_{L^{\infty}\left(R_{l}\right)}\right\} \\
& \leq C|T|^{\frac{1}{p}} \sum_{|\gamma+\beta|<k}\left\{\frac{\ell\left(R^{l}\right)^{|\gamma|}}{\left|S^{l+1}\right|^{1 / p}} \| D^{\beta+\gamma}\left(P\left(S^{l+1}\right)-P\left(S^{l+1} \cup S^{l}\right) \|_{L^{p}\left(S^{l+1}\right)}\right.\right. \\
& +\frac{\ell\left(R^{l}\right)^{|\gamma|}}{\left|S^{l}\right|^{1 / p}} \| D^{\beta+\gamma}\left(P\left(S^{l}\right)-P\left(S^{l+1} \cup S^{l}\right) \|_{L^{p}\left(S^{l}\right)}\right\} \\
& \leq C \sum_{|\gamma+\beta|<k} \ell_{n}(\tau(T))^{|\gamma|} \ell\left(S^{l}\right)^{k-|\beta|-|\gamma|}\left\|\nabla^{k} f\right\|_{L^{p}\left(S^{l} \cup S^{l+1}\right)} .
\end{aligned}
$$


Consequently,

$$
\begin{aligned}
\mathrm{I} & \leq C \sum_{T_{j} \cap T \neq \varnothing} \sum_{\beta \leq \alpha} \frac{1}{\ell(T)^{|\alpha|-|\beta|}} \sum_{l=1}^{M-1} \sum_{|\gamma+\beta|<k} \ell_{n}(\tau(T))^{|\gamma|} \ell\left(S^{l}\right)^{k-|\beta|-|\gamma|}\left\|\nabla^{k} f\right\|_{L^{p}\left(S^{l} \cup S^{l+1}\right)} \\
& \leq C \ell(T)^{k-|\alpha|}\left(\frac{\ell_{n}(\tau(T))}{\ell(T)}\right)^{k-1} \sum_{l=1}^{M-1}\left\|\nabla^{k} f\right\|_{L^{p}\left(S^{l} \cup S^{l+1}\right)} .
\end{aligned}
$$

Applying the Hölder inequality gives

$$
\mathrm{I} \leq C \ell(T)^{k-|\alpha|}\left(\frac{\ell_{n}(\tau(T))}{\ell(T)}\right)^{k-1} M^{1 / p^{\prime}}\left\|\nabla^{k} f\right\|_{L^{p}(\cup \mathscr{F}(T))},
$$

where $1 / p+1 / p^{\prime}=1$. But $M$ is the number of cubes in the chain joining $T^{*}$ and $T_{j}^{*}$, which we saw could be as large as $\ell_{n}(\tau(T)) / \ell(T)$, and then

$$
\mathrm{I} \leq C \ell(T)^{k-|\alpha|}\left(\frac{\ell_{n}(\tau(T))}{\ell(T)}\right)^{k-1 / p}\left\|\nabla^{k} f\right\|_{L^{p}(\cup \mathscr{F}(T))}
$$

II could be bounded by means of the same ideas.

A proposition equivalent to Proposition 4.16 can now be easily proved.

Proposition 4.19. If we set $\sigma(x)=(\ell(S(|x|)) /|x|)^{k}$, then

$$
\left\|\sigma D^{\alpha} \Lambda_{2} f\right\|_{L^{p}\left(\cup W_{4}\right)} \leq C\|f\|_{W^{k, p}(\cup \varphi)} .
$$

Proof. As we did in Proposition 4.16, let us observe that the weight $\sigma$ could be considered constant in each cube $T \in \mathcal{W}_{4}, \sigma_{T} \sim\left(\ell\left(S\left(z_{T}\right)\right) / z_{T}\right)^{k}$. Then $\left\|\sigma D^{\alpha} \Lambda_{2} f\right\|_{L^{p}\left(\cup^{o} W_{4}\right)}^{p}$

$$
\begin{aligned}
& =\sum_{T \in W_{4}}\left\|\sigma D^{\alpha} \Lambda_{2} f\right\|_{L^{p}(T)}^{p} \\
& \leq C \sum_{T \in W_{4}}\left(\frac{\ell\left(S\left(z_{T}\right)\right)}{z_{T}}\right)^{k p}\left\|D^{\alpha} \Lambda_{2} f\right\|_{L^{p}(T)}^{p} \\
& \leq C \sum_{T \in \mathscr{W}_{4}}\left(\frac{\ell\left(S\left(z_{T}\right)\right)}{z_{T}}\right)^{k p} \ell(T)^{(k-|\alpha|) p}\left(\frac{\ell_{n}(\tau(T))}{\ell(T)}\right)^{k p-1}\|f\|_{W^{k, p}(\cup \mathscr{F}(T))}^{p} \\
& =C \sum_{S \in \mathscr{S}} \sum_{T: \mathscr{F}(T) \ni S}\left(\frac{\ell\left(S\left(z_{T}\right)\right)}{z_{T}}\right)^{k p} \ell(T)^{(k-|\alpha|) p}\left(\frac{\ell_{n}(\tau(T))}{\ell(T)}\right)^{k p-1}\|f\|_{W^{k, p}(S)}^{p} .
\end{aligned}
$$

Now, observe that if we fix a cube $S \in \mathscr{Y}$, every cube $T \in \mathscr{W}_{4}$ such that $S \in \mathscr{F}(T)$ satisfies $\ell(T) \sim \ell(S)$ and $\ell_{n}(\tau(T)) \leq z_{S}$. By considering this and $|\alpha| \leq k$, we obtain

$$
\left\|\sigma D^{\alpha} \Lambda_{2} f\right\|_{L^{p}\left(\cup W_{4}\right)}^{p} \leq C \sum_{S \in \mathscr{S}} \sum_{T: \mathscr{F}(T) \ni S}\left(\frac{\ell\left(S\left(z_{T}\right)\right)}{z_{T}}\right)^{k p}\left(\frac{z_{S}}{\ell(T)}\right)^{k p-1}\|f\|_{W^{k, p}(S)}^{p} .
$$


Now, using property (2-12) we obtain

$$
\begin{aligned}
\left\|\sigma D^{\alpha} \Lambda_{2} f\right\|_{L^{p}\left(\cup^{\left.W_{4}\right)}\right.}^{p} & \leq C \sum_{S \in \mathscr{Y}} \sum_{T: \mathscr{F}(T) \ni S}\left(\frac{\ell(S)}{z_{S}}\right)^{k p}\left(\frac{z_{S}}{\ell(T)}\right)^{k p-1}\|f\|_{W^{k, p}(S)}^{p} \\
& \leq C \sum_{S \in \mathscr{S}} \sum_{T: \mathscr{F}(T) \ni S} \frac{\ell(S)}{z_{S}}\|f\|_{W^{k, p}(S)}^{p} .
\end{aligned}
$$

But, for a fixed $S \in \mathscr{Y}$, the number of cubes $T$ such that $S \in \mathscr{F}(T)$ is at most $C z_{S} / \ell(S)$, and then

$$
\begin{aligned}
\left\|\sigma D^{\alpha} \Lambda_{2} f\right\|_{L^{p}\left(\cup W_{4}\right)}^{p} & \leq C \sum_{S \in \mathscr{S}} \frac{z_{S}}{\ell(S)} \frac{\ell(S)}{z_{S}}\|f\|_{W^{k, p}(S)}^{p} \\
& \leq C \sum_{S \in \mathscr{S}}\|f\|_{W^{k, p}(S)}^{p}=\|f\|_{W^{k, p}(\cup \varphi)}^{p} .
\end{aligned}
$$

Remark 4.20. If $k p \neq 1$, property (2-12) is not necessary. Using $\ell\left(S\left(z_{T}\right)\right) \leq \ell(T)$, an argument similar to the one given in Proposition 4.16 can be applied: at the end of the proof of Proposition 4.16 the edges of all cubes $T$ such that $S \subset S(T)$ are classified in terms of $\ell(S)$. In the last part of the proof of Proposition 4.19, the same idea can be used in order to classify $z_{T}$ for all cubes $T$ such that $S \in \mathscr{F}(T)$. When $k p \neq 1$ the summation is bounded and the result follows.

Third stage. This stage is devoted to defining our extension operator in the cubes of $W_{5}$. We explain the construction of the reflected sets for each version of the extension, but we do not enter into details since the ideas are exactly the same as in Lemmas 4.14 and 4.18 and Propositions 4.16 and 4.19, according to the case.

For the first (dimensional) version of the extension, let us define

$$
S(U)=\bigcup\left\{S_{i}: \ell(U) \leq z_{i}<2 \ell(U)\right\} .
$$

It is clear that $d(U, S(U)) \leq C \ell(U)$. On the other hand, $S(U)$ is a tower of cubes that admits an interior rectangle $R^{1}$, with $\ell_{i}\left(R^{1}\right) \sim \ell(S(\ell(U)))$ for $i=1, \ldots, n-1$ and $\ell_{n}\left(R^{1}\right) \sim \ell(U)$. Because of property (2-13), there is an exterior rectangle $R^{2} \supset S(U)$ such that $R^{2} \sim R^{1}$. Hence Remark 4.13 holds for cubes in $\mathcal{W}_{5}$, and so do Lemma 4.14 and Proposition 4.16. As we did earlier, we define $P_{U_{j}}=P\left(R_{j}^{1}\right)$. The last thing to notice is that if $T \in \mathscr{W}_{4}$ and $U \in W_{5}$ are such that $T \cap U \neq \varnothing$, then $d(S(U), S(T)) \leq C \ell(T)$, and then there is a finite chain of towers that joins $S(U)$ and $S(T)$. This guarantees that the transition between $\mathscr{W}_{4}$ and $\mathscr{W}_{5}$ is smooth.

For the second (derivative) version, let us define $U^{*}=S_{i}$, the cube in $\mathscr{Y}$ with $i$ the maximum index such that $\ell\left(S_{i}\right) \geq \ell(U)$. This implies $\ell\left(U^{*}\right) \sim \ell(U)$, which is the essential property of the reflected cube in this case. On the other hand, $d\left(U, U^{*}\right) \leq C z_{U^{*}}$. Once again, we define $P_{U_{j}}=P\left(U_{j}^{*}\right)$. It is clear that if $T \in \mathcal{W}_{4}$ and $U \in W_{5}$ are such that $T \cap U \neq \varnothing$ and $U^{*} \sim T^{*}$, then $d\left(U^{*}, T^{*}\right) \leq C z_{T^{*}}$, so 
the norm of the extension can be bounded in the frontier between $\mathscr{W}_{4}$ and $W_{5}$ as we did in $W_{4}$.

As we did in the previous sections, let us define, for both versions,

$$
\Lambda_{3} f(x)=\sum_{U_{j} \in W_{5}} P_{U_{j}}(x) \psi_{j}(x)
$$

The last matter that we need to deal with is the superposition induced by this definitions of reflected sets. In the previous stage we introduced $\widetilde{W}_{4}$ in order to help us count some sets of cubes in $\mathscr{W}_{4}$. Similarly, we now introduce $\widetilde{W}_{5}=\mathscr{W}\left(\mathbb{R}^{n} \backslash\{0\}\right)$. Thanks to Remark 2.4 we may define, for every $U \in \mathcal{W}_{5}, \widetilde{U}$ the cube in $\widetilde{W}_{5}$ such that $U \subset \widetilde{U}$. On the other hand the ideas exposed earlier (see Lemma 4.11) lead us to conclude that $U \sim \widetilde{U}$. The number of cubes in $\widetilde{W}_{5}$ with edges of a certain length $2^{-l}$ is bounded by a constant depending only on the dimension $n$. The same holds for cubes in $\mathscr{W}_{5}$. Consequently, after this third stage, every cube in $\mathscr{Y}$ is loaded with at most a bounded quantity of cubes of the exterior of $\Omega$.

Our complete extension operator is then

$$
\Lambda f(x)=\Lambda_{1} f(x)+\Lambda_{2} f(x)+\Lambda_{3} f(x) .
$$

For every $x=\left(x^{\prime}, x_{n}\right) \in \mathscr{W}_{4}$, we have $x_{n} \sim|x|$. We use this fact to write the weight in terms of $|x|$ instead of $x_{n}$. Since the third term of the extension is also radial, the weight can be taken as

$$
\left(\frac{\ell(S(|x|))}{|x|}\right)^{\gamma}
$$

where $\gamma$ is the exponent corresponding to the case in question.

Except for a few technical details that are treated in Section 6, the proof of Theorem 4.1 is concluded.

\section{Curved cusps}

Theorem 5.1. Let $\Omega \subset \mathbb{R}^{n}$ be a domain with an external curved cusp at the origin.

(a) If the spine $\mathscr{Y}$ satisfies (2-12), there is an extension operator

$$
\Lambda: W^{k, p}(\Omega) \rightarrow W_{\sigma}^{k, p}\left(\mathbb{R}^{n}\right),
$$

where

$$
\sigma(x)=\left\{\begin{array}{cl}
1 & \text { if } x \in \Omega, \\
\left(\frac{\ell(S(|x|))}{|x|}\right)^{k} & \text { if } x \in \Omega^{c} .
\end{array}\right.
$$


(b) If the spine 9 satisfies (2-13), there is an extension operator

$$
\Lambda: W^{k, p}(\Omega) \rightarrow W_{\sigma}^{k, p}\left(\mathbb{R}^{n}\right),
$$

where

$$
\sigma(x)=\left\{\begin{array}{cl}
1 & \text { if } x \in \Omega, \\
\left(\frac{\ell(S(|x|))}{|x|}\right)^{(n-1) / p} & \text { if } x \in \Omega^{c} .
\end{array}\right.
$$

(c) In either case (a) or (b), assuming (2-13) holds, if $\tilde{\sigma}$ is such that there is an extension operator $\tilde{\Lambda}: W^{k, p}(\Omega) \rightarrow W_{\tilde{\sigma}}^{k, p}\left(\mathbb{R}^{n}\right)$, then

$$
\tilde{\sigma}(x) \leq C \sigma(x) \text { for all } x \in U \backslash \Omega \text {. }
$$

We only sketch a proof of Theorem 5.1. We introduce a stage zero, consisting of an extension of a curved cusp $\Omega$ to a larger domain that includes a normal cusp $\widehat{\Omega}$. Functions defined on $\widehat{\Omega}$ will be extended as in Theorem 4.1. The most important fact to mention is that after stage one, the distance from cubes in $W_{4}$ and $W_{5}$ to $\Omega$ is comparable with the distance from them to $\widehat{\Omega}$, and so will be the weights.

Stage zero. Let $\Omega$ be a domain satisfying Definition 2.7, and let $\mathscr{Y}=\left\{S_{i}\right\}_{i=1}^{\infty}$ be its spine. Then $d\left(S_{i}, \hat{x_{n}}\right) \leq C_{\Omega} \ell\left(S_{i}\right)$, and we may take $C_{\Omega} \geq K$. Assuming $\ell\left(S_{i}\right) \leq 1$, let us consider

$$
\widetilde{\Omega}=\bigcup_{i} 4\left(C_{\Omega}+1\right) S_{i}
$$

Clearly, $\Omega \subset \bigcup_{i} C_{\Omega} S_{i}$. Even more: Let us take $S_{i}^{\prime}$ to be the translation of $S_{i}$ to the axis $x_{n}$, so $S_{i}^{\prime} \cap S_{i+1}^{\prime}=F_{S_{i+1}^{\prime}}^{u}$ and $z_{S_{i}^{\prime}}=z_{S_{i}}$. Then, if we set $\widehat{\Omega}=\bigcup_{i} 2 C_{\Omega} S_{i}^{\prime}$, we have

$$
\Omega \subset \widehat{\Omega} \subset \widetilde{\Omega}
$$

Lemma 4.6 can be reproduced in order to find a reflected cube for every $Q$ in $W_{2}$ such that $Q \subset \widetilde{\Omega}$, by just changing $K$ for $4\left(C_{\Omega}+1\right)$. Consequently, a first (unweighted) extension can be performed as in stage one. Let us denote by $\Lambda_{0} f$ the extension of $f$ to $\widetilde{\Omega}$, and let us take $\widehat{f}: \widehat{\Omega} \rightarrow \mathbb{R}, \widehat{f}=\left.\Lambda_{0} f\right|_{\widehat{\Omega}}$. Observe that $\widehat{\Omega}$ is a normal cusp. Then, we can extend $\widehat{f}$ as in Theorem 4.1. Let us denote by $\widehat{W}_{3}, \widehat{W}_{4}, \widehat{W}_{5}$, the subsets of the Whitney decomposition of the exterior of $\widehat{\Omega}$ corresponding to stage one, two and three respectively. If we denote by $\widehat{\mathscr{S}}=\left\{\widehat{S}_{i}\right\}_{i}$ the spine of $\widehat{\Omega}$ (observe that $\widehat{S}_{i}$ is not necessarily $S_{i}^{\prime}$, but they are equivalent), we have $\widehat{S}_{i} \sim S_{i}$. Now, if we take $T \in \widehat{\mathscr{W}}_{4}$, such that $z_{i} \leq z_{T}<z_{i-1}$, then

$$
\ell(T) \geq C \ell\left(\widehat{S}_{i}\right) \geq C \ell\left(S_{i}\right) .
$$


Furthermore,

$$
\begin{gathered}
d\left(T, S_{i}\right) \leq C d\left(T, \widehat{S}_{i}\right) \leq C \ell(T), \\
d(T, \partial \widehat{\Omega}) \leq d(T, \partial \Omega) \leq C d\left(T, \widehat{S}_{i}\right) \leq C d(T, \partial \widehat{\Omega}) .
\end{gathered}
$$

The weight of the extension operator based on $\widehat{\Omega}$ is expressed in terms of $\widehat{S}_{i}$, but these inequalities allow us to change it for $S_{i}$, and then Theorem 5.1 is proved by using the results given in the next section.

\section{Density of $C^{\infty}$ functions}

Following Jones we prove that certain set of regular functions is dense in $W^{k, p}(\Omega)$.

Proposition 6.1 [Jones 1981]. Let $D$ be a $\varepsilon$-uniform domain, and $f \in W^{k, p}(D)$. For every $\eta>0$ there is a function $g \in C^{\infty}\left(\mathbb{R}^{n}\right)$ such that $\|f-g\|_{W^{k, p}(D)}<\eta$.

Remark 6.2. The proof of this result relies on the existence of certain chain of dyadic cubes constructed in [Jones 1981] by means of properties (2-1) and (2-2). Similar arguments can be carried out using properties (2-6) and (2-7) instead of (2-1) and (2-2). In this way Proposition 6.1 can be proved for certain sets $\check{\Omega}_{i} \subset$ $\Omega_{i} \cup \Omega_{i+1}$ defined below.

Theorem 6.3. Let $\Omega$ be a cusp, and $f \in W^{k, p}(\Omega)$. Given $\eta>0$, there is a function $g \in C^{\infty}\left(\mathbb{R}_{+}^{n}\right)$ such that $\|f-g\|_{W^{k, p}(\Omega)}<C \eta$.

Proof. Let us define

$$
\begin{aligned}
& \check{\Omega}_{i}=\Omega \cap\left\{x=\left(x^{\prime}, x_{n}\right): z_{i}-\frac{\ell\left(S_{i+1}\right)}{2} \leq x_{n}<z_{i-1}+\frac{\ell\left(S_{i-1}\right)}{2}\right\}, \\
& \check{\Omega}_{i}^{\prime}=\Omega \cap\left\{x=\left(x^{\prime}, x_{n}\right): z_{i}-\frac{\ell\left(S_{i+1}\right)}{2} \leq x_{n}<z_{i}+\frac{\ell\left(S_{i}\right)}{2}\right\} .
\end{aligned}
$$

Let us consider $g_{i} \in C^{\infty}$ such that $\left\|f-g_{i}\right\|_{W^{k, p}\left(\check{\Omega}_{i}\right)}<\left(\eta / 2^{i}\right) \ell\left(S_{i}\right)^{k}$. Observe that the existence of such a function is guaranteed by Proposition 6.1 and Remark 6.2.

Let $\left\{\psi_{i}\right\}_{i}$ a partition of unity such that

$$
\psi_{i} \in C_{0}^{\infty}\left(\left[z_{i}-\ell\left(S_{i+1}\right) / 2, z_{i-1}+\ell\left(S_{i-1}\right) / 2\right]\right),
$$

$\sum \psi_{i}(t) \equiv 1$ for all $t \in\left(0, z_{1}\right]$, and $\left|D^{r} \psi_{i}\right| \leq C / \ell\left(S_{i}\right)^{r}$.

Let us define

$$
g(x)=\sum_{i=2}^{\infty} g_{i}(x) \psi_{i}\left(x_{n}\right) .
$$

Observe that, in $\check{\Omega}_{i}^{\prime}, \psi_{i}+\psi_{i+1} \equiv 1$. Then

$$
\begin{aligned}
\left\|D^{\alpha}(f-g)\right\|_{L^{p}\left(\check{\Omega}_{i}^{\prime}\right)} & \leq\left\|D^{\alpha}\left(f-\left(\psi_{i} g_{i}+\psi_{i+1} g_{i+1}\right)\right)\right\|_{L^{p}\left(\check{\Omega}_{i}^{\prime}\right)} \\
& \left.\leq\left\|D^{\alpha}\left(\psi_{i}\left(f-g_{i}\right)\right)\right\|_{L^{p}\left(\check{\Omega}_{i}^{\prime}\right)}+\left\|D^{\alpha}\left(\psi_{i+1}\left(f-g_{i+1}\right)\right)\right\|_{L^{p}\left(\check{\Omega}_{i}^{\prime}\right.}\right)
\end{aligned}
$$


But

$$
\begin{aligned}
& \left\|D^{\alpha}\left(\psi_{i}\left(f-g_{i}\right)\right)\right\|_{L^{p}\left(\check{\Omega}_{i}^{\prime}\right)} \leq\left\|\sum_{\beta \leq \alpha} D^{\alpha-\beta} \psi_{i} D^{\beta}\left(f-g_{i}\right)\right\|_{L^{p}\left(\check{\Omega}_{i}^{\prime}\right)} \\
& \leq \sum_{\beta \leq \alpha} \frac{C}{\ell\left(S_{i}\right)^{|\alpha|-|\beta|}}\left\|D^{\beta}\left(f-g_{i}\right)\right\|_{L^{p}\left(\check{\Omega}_{i}^{\prime}\right)} \\
& \leq \frac{C}{\ell\left(S_{i}\right)^{|\alpha|-|\beta|}} \frac{\eta}{2^{i}} \ell\left(S_{i}\right)^{k} \leq C \frac{\eta}{2^{i}} .
\end{aligned}
$$

Consequently

$$
\|f-g\|_{W^{k, p}(\Omega)}^{p}=\sum_{i=1}^{\infty}\|f-g\|_{W^{k, p}\left(\check{\Omega}_{i}^{\prime}\right)}^{p} \leq \sum_{i=1}^{\infty} C \frac{\eta^{p}}{2^{p i}} \leq C \eta^{p} .
$$

Following Jones's arguments one can show that $D^{\alpha} \Lambda f=D^{\alpha} f \chi_{\bar{\Omega}}+D^{\alpha} \Lambda f \chi_{\bar{\Omega}^{c}}$ is in $\operatorname{Lip}_{\text {loc }}\left(\mathbb{R}^{n} \backslash\{0\}\right)$ for all $\alpha,|\alpha| \leq k-1$, and for any $f \in C^{\infty}\left(\mathbb{R}_{+}^{n}\right)$. Indeed, taking into account that the first stage of our extension process follows Jones's reflection method, we can reproduce the ideas of Lemma 3.5 in [Jones 1981]. The boundedness of $f$ in the arguments given in that reference is crucial: here we should use the fact that $f$ is bounded in any compact set $K$ such that $0 \notin K$.

Recalling that $|\partial \Omega|=0$ we see that Theorems 4.1 and 5.1 are proved by using density arguments and Theorem 6.3.

\section{The weighted case}

For all measurable sets $S \subset \mathbb{R}^{n}$, let $\omega(S)$ be the measure induced by the weight $\omega$,

$$
\omega(S)=\int_{S} \omega
$$

We say $\omega$ is doubling if for every cube $Q \in \mathbb{R}^{n}, \omega(2 Q) \leq C \omega(Q)$ with $C$ independent of $Q$.

Chua [1992; 1994] adapts Jones's techniques for proving an extension theorem for locally uniform domains in the weighted case. We state a version of his results.

Theorem B. Let $\Omega$ be an $(\varepsilon, \delta)$ connected domain, $1 \leq p<\infty$. Suppose that $\omega$ is doubling, $\omega^{-1 /(p-1)}$ is locally integrable and $\operatorname{Lip}_{\text {loc }}^{k-1} \mathbb{R}^{n}$ is dense on $W_{\omega}^{k, p}(\Omega)$. Finally, suppose that for every cube $Q$ and every $f \in \operatorname{Lip}_{\text {loc }} \mathbb{R}^{n}$,

$$
\left\|f-f_{Q, \omega}\right\|_{L_{\omega}^{p}(Q)} \leq C \ell(Q)\|\nabla f\|_{L_{\omega}^{p}(Q)},
$$

where $f_{Q, \omega}=\int f d \omega / \omega(Q)$. Then an extension operator $\Lambda: W_{\omega}^{k, p}(\Omega) \rightarrow W_{\omega}^{k, p}\left(\mathbb{R}^{n}\right)$ exists.

Property (7-1) is just a weighted Poincaré inequality. A simpler but stronger hypothesis, that implies all the requirements on the weight, is that $\omega \in A_{p}$, the 
class of weights satisfying Muckenhoupt's condition

$$
\sup _{\text {cubes } Q \subset \mathbb{R}^{n}} \frac{1}{|Q|}\left(\int_{Q} \omega(x) d x\right)\left(\int_{Q} \omega(x)^{-1 /(p-1)} d x\right)^{p-1} \leq C<\infty .
$$

Chua's extension operator is constructed as Jones's one: For each cube $Q_{j} \in W^{c}$ near the domain, a reflected cube $Q_{j}^{*} \in \mathcal{W}$ is found (as in Lemma 4.6). Given $f \in W_{\omega}^{k, p}(\Omega)$, a suitable polynomial $P_{j}=P\left(Q_{j}^{*}, \omega\right)$, that can be constructed thanks to (7-1), is associated to $Q_{j}$. Hence the operator is the smooth summation of all the $\left\{P_{j}\right\}_{j}$.

The doubling condition is crucial for Chua's arguments to hold. Indeed, since $d\left(Q, Q^{*}\right) \leq C \ell(Q)$, a bounded expansion of $Q, \widetilde{Q}=c Q$, contains both cubes $Q$ and $Q^{*}$. But $\omega$ being doubling, $\omega(\widetilde{Q}) \leq C \omega(Q)$. This allows a comparison between the values of the weight $\omega$ over $Q$ and over $Q^{*}$. Therefore, the weighted norm of the extension in $Q$ can be bounded by the weighted norm of the function in $Q^{*}$ just as in Lemma 3.2 in [Jones 1981] or Lemma 4.10 in this paper.

Since the first stage of our extension process agrees with that of uniform domains, Chua's techniques could be applied. However, the second stage presents a very different situation. Reflected sets for cubes in $\mathcal{W}_{4}$ do not fulfill properties (4-4) and (4-5) of Jones's reflected cube. In the dimensional-horizontal version, the reflected set of $Q$ is a rectangle $S(Q)$, not a cube, and whereas $d(Q, S(Q)) \sim \ell(Q)$, the edges $\ell_{i}(S(Q))$ are not equivalent to $\ell(Q)$, so (4-4) fails. Consequently, the values of the weight $\omega$ over $Q$ cannot be estimated by its values over $S(Q)$. On the other hand, in the derivative-vertical version, the reflected set of $Q$ is a cube $Q^{*}$, with $\ell\left(Q^{*}\right) \sim \ell(Q)$, but it may happen that $d\left(Q, Q^{*}\right) \gg \ell(Q)$, so (4-5) fails. In this case, no bounded fixed expansion of $Q$ could reach $Q^{*}$, and the doubling property of $\omega$ is useless.

However, it is noteworthy that some particular weights can be easily integrated into our extension process. We present here two examples involved in several applications: weights depending on the distance to the boundary of $\Omega$, that fit easily with the derivative version of the extension, and weights depending on the distance to 0 (the tip of the cusp), that are naturally adapted to the dimensional version.

We analyze each type of weight separately.

Weights depending on $d(x, 0)=|x|$-the dimensional case. Observe that near the origin we have that $|x| \sim x_{n}$, for all $x \in \Omega$. Moreover, the same thing holds close enough to $\Omega$, and in particular in the sets $\bigcup \mathcal{W}_{3}$ and $\bigcup \mathcal{W}_{4}$. Let $\omega: \mathbb{R}^{n} \rightarrow \mathbb{R}$, $\omega \geq 0$, be a radial weight. We set $\omega(x)=\hat{\omega}(|x|)$, and we assume that $\hat{\omega}: \mathbb{R}_{\geq 0} \rightarrow \mathbb{R}_{\geq 0}$ is a monotonic function that satisfies $\hat{\omega}(2 t) \sim \hat{\omega}(t)$. Notice that the only interesting case is that either $\hat{\omega}(t) \rightarrow 0$ or $\hat{\omega}(t) \rightarrow \infty$ when $t \rightarrow 0$, since otherwise the weighted 
space agrees with the already treated case of $W^{k, p}$. Let us mention that $W_{\omega}^{k, p}$ is a Banach space [Kufner 1985] for any open set $\Omega$.

Remark 7.1. Observe that for every $x, \tilde{x} \in Q \in \mathcal{W}_{3} \cup \mathcal{W}_{4}$, we have $\omega(x) \sim \omega(\tilde{x})$, therefore we can pick a constant $\omega_{Q}$ such that $\omega(x) \sim \omega_{Q}$ for all $x \in Q \in \mathscr{W}_{3} \cup \mathcal{W}_{4}$.

This fact is the key tool for our weighted extension process. We detail the proof of the first stage: Lemma 4.6 guarantees that $d\left(Q, Q^{*}\right) \leq C \ell(Q)$, for all $Q \in W_{3}$. This implies $z_{Q^{*}} \sim z_{Q}$, and consequently $\omega_{Q} \sim \omega_{Q^{*}}$. Furthermore, it is easy to see that $\omega_{Q} \sim \omega_{S}$ for every $S \in \mathscr{F}(Q)$.

Lemma 7.2. If $Q \in \mathcal{W}_{3}$ is far from $\mathcal{W}_{4}$, then

$$
\left\|D^{\alpha} \Lambda f\right\|_{L_{\omega}^{p}(Q)} \leq C\left\{\ell(Q)^{k-|\alpha|}\left\|\nabla^{k} f\right\|_{L_{\omega}^{p}(\mathscr{F}(Q))}+\|f\|_{W_{\omega}^{k, p}\left(Q^{*}\right)}\right\} .
$$

Proof. Just apply the constant approximation of the weight and Lemma 4.10, so

$$
\begin{aligned}
\left\|D^{\alpha} \Lambda f\right\|_{L_{\omega}^{p}(Q)} & =\left\|\omega D^{\alpha} \Lambda f\right\|_{L_{p}(Q)} \leq C \omega_{Q}\left\|D^{\alpha} \Lambda f\right\|_{L^{p}(Q)} \\
& \leq C \omega_{Q}\left\{\ell(Q)^{k-|\alpha|}\left\|\nabla^{k} f\right\|_{L^{p}(\mathscr{F}(Q))}+\|f\|_{W^{k, p}\left(Q^{*}\right)}\right\} \\
& \leq C\left\{\ell(Q)^{k-|\alpha|}\left\|\nabla^{k} f\right\|_{L_{\omega}^{p}(\mathscr{F}(Q))}+\|f\|_{W_{\omega}^{k, p}\left(Q^{*}\right)}\right\} .
\end{aligned}
$$

For the second stage we use essentially the same idea: the weight, being approximately constant over every cube, can be pulled in or out of integrals, so the weighted norm can be estimated using the nonweighted lemmas of Section 4.

It is clear that $\omega_{T} \sim \omega_{S(T)}$, for all $T \in \mathscr{W}_{4}$. The weighted form of Lemma 4.14 can be proved exactly as Lemma 7.2, so the next proposition follows, completing the second stage for this version.

Proposition 7.3. If we set $\sigma(x)=(\ell(S(|x|)) /|x|)^{(n-1) / p}$, then

$$
\left\|\sigma D^{\alpha} f\right\|_{L_{\omega}^{p}\left(W_{4}\right)} \leq C\|f\|_{W_{\omega}^{k, p}(\mathscr{S})} .
$$

Exactly the same ideas can be used for the third stage.

Weights depending on $d_{\partial \Omega}-$ the derivative case. We obviously have

$$
d(x, \partial \Omega) \sim \ell(Q) \text { for all } x \in Q \text { and all } Q \in \mathcal{W} \cup \mathcal{W}^{c} \text {. }
$$

Let $\hat{\omega}: \mathbb{R}_{+} \rightarrow \mathbb{R}_{+}$be a monotonic function such that $\hat{\omega}(2 t) \sim \hat{\omega}(t)$, and let $\omega: \mathbb{R}^{n} \rightarrow \mathbb{R}^{n}$ be the weight $\omega(x)=\hat{\omega}\left(d_{\partial \Omega}(x)\right)$. In this case this implies that $\omega$ can be taken as a constant $\omega_{Q}$ over every cube $Q \in \mathscr{W} \cup W^{c}$.

This leads us to the following corollary of Lemma 4.10 (whose proof is exactly as the one of Lemma 7.2).

Lemma 7.4. Let $\Omega$ be a domain satisfying Definition 2.6. Then

$$
\left\|D \Lambda_{1} f\right\|_{L_{\omega}^{p}(Q)} \leq C\left\{\ell(Q)^{k-|\alpha|}\left\|\nabla^{k} f\right\|_{L_{\omega}^{p}(\mathscr{F}(Q))}+\left\|D^{\alpha} f\right\|_{L_{\omega}^{p}\left(Q^{*}\right)}\right\} .
$$


For the second stage, let us recall that in the derivative version, $\ell(Q) \sim \ell\left(Q^{*}\right)$, and then $d_{\partial \Omega}(Q) \sim d_{\partial \Omega}\left(Q^{*}\right)$. This fact is enough to complete the second stage.

Once again, the third stage follows in the same way.

Theorem 7.5. Let $\Omega \subset \mathbb{R}^{n}$ be a domain with an external normal cusp at the origin. Let $\hat{\omega}: \mathbb{R}_{+} \rightarrow \mathbb{R}_{+}$be a monotonic function satisfying $\hat{\omega}(2 t) \sim \hat{\omega}(t)$, and $1 \leq p<\infty$.

(a) Consider the weighted Sobolev space $W_{\omega}^{k, p}(\Omega)$, where $\omega(x)=\hat{\omega}\left(d_{\partial \Omega}(x)\right)$ for all $x$. Suppose that $C^{\infty}\left(\bar{\Omega} \cap \mathbb{R}_{+}^{n}\right) \cap W_{\omega}^{k, p}(\Omega)$ is dense in $W_{\omega}^{k, p}(\Omega)$ (see Remark 7.7 below) and that $\omega^{-1 /(p-1)}$ is locally integrable. If the spine $\mathscr{Y}$ satisfies (2-12), there exists an extension operator

$$
\Lambda: W_{\omega}^{k, p}(\Omega) \rightarrow W_{\omega \sigma}^{k, p}\left(\mathbb{R}^{n}\right)
$$

where

$$
\sigma(x)=\left\{\begin{array}{cl}
1 & \text { if } x \in \Omega, \\
\left(\frac{\ell(S(|x|))}{|x|}\right)^{k} & \text { if } x \in \Omega^{c} .
\end{array}\right.
$$

(b) Consider the weighted Sobolev space $W_{\omega}^{k, p}(\Omega)$, where $\omega(x)=\hat{\omega}(|x|)$ for all $x$. If the spine $\mathcal{Y}$ satisfies (2-13), there exists an extension operator

$$
\Lambda: W_{\omega}^{k, p}(\Omega) \rightarrow W_{\omega \sigma}^{k, p}\left(\mathbb{R}^{n}\right)
$$

where

$$
\sigma(x)=\left\{\begin{array}{cl}
1 & \text { if } x \in \Omega, \\
\left(\frac{\ell(S(|x|))}{|x|}\right)^{(n-1) / p} & \text { if } x \in \Omega^{c} .
\end{array}\right.
$$

Definition 7.6. For $0 \leq m \leq n$, a set $F$ is called $m$-regular, if there exists a positive constant $C$ such that

$$
C^{-1} r^{m}<\mathscr{H}^{m}(B(x, r))<C r^{m},
$$

for all $x \in F$ and $0<r \leq \operatorname{diam} F$. Here $\mathscr{H}^{m}$ stands for the $m$ dimensional Hausdorff measure and the restriction $0<r \leq \operatorname{diam} F$ is eliminated if $F$ is a set with only one point.

Let us mention that some self similar fractals, such as the well known Koch curve, are $m$-regular with $m \notin \mathbb{N}$ (in fact $m=\log (4) / \log (3)$ in the Koch example).

Remark 7.7. As we stated above, for a uniform domain $D$, a general and simple condition that guarantees the density of $C^{\infty}(\bar{D})$ in $W_{\omega}^{k, p}(D)$ is that $\omega \in A_{p}$ (see [Chua 1992]). Under extra assumptions on the boundary of $D$ it is possible to find conditions under which weights of the type $d(\cdot, \partial D)^{\mu}$ belong to $A_{p}$. Indeed, that holds for $-(n-m)<\mu<(n-m)(p-1)$, provided that $\partial D$ is a compact set contained in an $m$-regular set (see [Durán and López García 2010a]). In this case 
we can replicate Theorem 6.3 by using a weighted version of Proposition 6.1 and arguing along the lines given in Remark 6.2 (using Chua's results). Therefore the density assumption in item (a) of Theorem 7.5 can be removed.

Let us observe that for a "good" domain $D$, one expects $m=n-1$, therefore the range $-1<\mu<p-1$ is precisely the one for which the extension problem makes sense and it is nontrivial. Indeed, on one hand if $\mu \geq p-1$, then $\omega^{-1 /(p-1)}$ is not in $L_{\text {loc }}^{1}\left(\mathbb{R}^{n}\right)$ and the weighted global space can not be defined in the standard way. On the other, taking for instance $D$ Lipschitz and $\mu \leq-1$ it can be shown that $C_{0}^{\infty}(D)$ is dense in $W_{\omega}^{k, p}(D)$ [Kufner 1985], and therefore functions in that space can be extended by 0 .

Remark 7.8. Let us notice that the density is not needed in item (b) since it can be obtained by using the arguments given in Theorem 6.3 and taking into account that the weight is essentially constant over every $\check{\Omega}_{i}$.

Theorem 7.5 can be reproduced for curved cusps, by means of an easy adaptation of stage zero.

\section{Examples and concluding remarks}

Below we show that every domain satisfying Definition A is an external cusp in the sense of Definition 2.7 (or Definition 2.6). On the other hand our results can be understood in the following way: the role of the "profile" function $\varphi$ given in Definition A can be relaxed in the sense that it can describe only the speed of the narrowing of $\Omega$ towards the origin (if the spine of $\Omega$ decreases as $\varphi, \ell(S(z)) \sim \varphi(z)$ ), provided that $\partial \Omega \backslash\{0\}$ remains smooth enough. Consequently, the weight $\sigma$ in Theorem 5.1 can be expressed as

$$
\sigma(x)=\left(\frac{\varphi(|x|)}{|x|}\right)^{\gamma}
$$

where $\gamma=k$ or $(n-1) / p$, depending on the case.

Let us notice that both extension procedures for Theorems 4.1 and 5.1 - in contrast to what happens in Theorem A - are independent of the relationship between $k, p$ and $n$. Consequently, we proved that hypothesis (1-3) (or its generalization, $(2-12))$ is not necessary in any case as long as (1-4) (or (2-13)) holds, and vice versa.

First, we present some domains that do not satisfy Definition A but are included in Definitions 2.6 or 2.7 .

The first simple example is given by

$$
\Omega=\left\{(x, y) \in \mathbb{R}^{2}: y^{3}<x<y^{2}\right\} .
$$


This domain does not satisfy Definition A, however, it is easy to see that it is an external curved cusp.

The second example is general, and we devote a few lines to it.

Definition 8.1. Let $\Omega \subset \mathbb{R}^{n}(n \geq 2)$ be a domain with compact boundary $\partial \Omega$. Assume that $0 \in \partial \Omega$. We say that $\Omega$ has a restricted external cusp at the origin if there exists a neighborhood $U \subset \mathbb{R}^{n}$ of 0 such that

$$
U \cap \Omega=\left\{(x, z) \in \mathbb{R}^{n-1} \times \mathbb{R}: x \in \varphi(z) \varpi\right\},
$$

where $\varpi \subset \mathbb{R}^{n-1}$ is a bounded uniform domain and $\varphi: \mathbb{R}^{+} \rightarrow \mathbb{R}^{+}$is a Lipschitz increasing function such that $\varphi(0)=0$ and $\varphi(t) / t \rightarrow 0\left(t \rightarrow 0^{+}\right)$.

It is clear that every outer peak satisfying Definition A is a restricted external cusp.

Claim 8.2. Every restricted cusp satisfies Definition 2.7 (or 2.6).

We sketch the proof of this claim through a series of observations.

Given $\Omega$ a restricted cusp, let us define $\Omega_{z}$ to be the set of points of $\Omega$ at height $z$, and the boundary of this set to be $\partial \Omega_{z}:=\left\{(x, z) \in \mathbb{R}^{n}: x \in \varphi(z) \partial \varpi\right\}$.

Observe that the distance from a point $(x, z) \in \Omega$ to $\partial \Omega$ is equivalent to its distance to $\partial \Omega_{z}$. Indeed, it is clear that $d((x, z), \partial \Omega) \leq d\left((x, z), \partial \Omega_{z}\right)$. On the other hand, let us set $x=\varphi(z) \zeta$, for some $\zeta \in \varpi$. Let $\left(x_{0}, z_{0}\right)=\left(\varphi\left(z_{0}\right) \zeta_{0}, z_{0}\right) \in \partial \Omega$ be such that $d((x, z), \partial \Omega)=d\left((x, z),\left(x_{0}, z_{0}\right)\right)$. Naturally, $\tilde{x}_{0}=\left(\varphi(z) \zeta_{0}, z\right)$ is in $\partial \Omega_{z}$. Then

$$
\begin{aligned}
d\left((x, z), \partial \Omega_{z}\right) \leq\left|x-\tilde{x}_{0}\right| & =\left|\varphi(z) \zeta-\varphi(z) \zeta_{0}\right| \\
& \leq\left|\varphi(z) \zeta-\varphi\left(z_{0}\right) \zeta_{0}\right|+\left|\varphi\left(z_{0}\right)-\varphi(z)\right|\left|\zeta_{0}\right| \\
& \leq\left|\varphi(z) \zeta-\varphi\left(z_{0}\right) \zeta_{0}\right|+C_{\varphi} C_{\varpi}\left|z_{0}-z\right| \\
& \leq C\left(\left|\varphi(z) \zeta-\varphi\left(z_{0}\right) \zeta_{0}\right|+\left|z_{0}-z\right|\right) \\
& \leq C d\left((x, z),\left(x_{0}, z_{0}\right)\right)=C d((x, z), \partial \Omega),
\end{aligned}
$$

where $C_{\varphi}$ is the Lipschitz constant of $\varphi$ and $C_{\varpi}=\sup \{\|\xi\|: \xi \in \varpi\}$.

Let $r_{\varpi}$ be the inner radius of $\varpi$ :

$$
r_{\varpi}=\sup _{x \in \varpi} \inf _{y \in \partial \varpi} d(x, y),
$$

and let $c_{\varpi} \in \varpi$ be a point such that $B\left(c_{\varpi}, r_{\varpi}\right) \subset \varpi$.

Let us consider the curve $\Gamma: \mathbb{R}_{+} \rightarrow \mathbb{R}^{n}, \Gamma(t)=\left(\varphi(t) c_{\varpi}, t\right)$, that describes the "center" of $\Omega$. Let $\widetilde{\mathscr{S}}$ be the set of all cubes $S \in \mathcal{W}=\mathscr{W}(\Omega)$ such that $S \cap \Gamma(t) \neq \varnothing$. Let $\mathscr{S}=\left\{S_{i}\right\}_{i=1}^{\infty}$ be a subset of $\widetilde{\mathscr{S}}$ such that $S_{i} \cap S_{i+1} \neq \varnothing$ and $z_{S_{i+1}}<z_{S_{i}}$ (this is possible because $\varphi(t) / t \rightarrow 0) . \mathscr{S}$ is the spine of $\Omega$. 
Since $\varphi$ is Lipschitz, we have

$$
\varphi(z+C \varphi(z))-\varphi(z) \leq C_{\varphi}(z+C \varphi(z)-z)=C \varphi(z) .
$$

Then

$$
\varphi(z+C \varphi(z)) \leq C \varphi(z) .
$$

On the other hand $d\left(\Gamma(t), \partial \Omega_{t}\right)=r_{\varpi} \varphi(t)$, and consequently $d(\Gamma(t), \partial \Omega) \sim \varphi(t)$. Taking this into account, (8-1) implies that $\ell\left(S_{i}\right) \sim \varphi\left(z_{i}\right)$.

Properties (2-10) and (2-11) (as well as (2-3) and (2-4) when $c_{\varpi}$ can be taken equal to 0 ) follow easily from the definition of $\mathscr{Y}$. The covering property (2-5) is a consequence of (8-1). Since $\varpi$ is a fixed bounded domain, there is a radius $R_{\varpi}$ such that $\varpi \subset B\left(c_{\varpi}, R_{\varpi}\right)$. This radius scaled to the section $\Omega_{z}$ is $\varphi(z) R_{\varpi}$, but $\varphi(z)$ is essentially the length $\ell(S(z))$. Taking (8-1) into consideration, this implies that there is a constant $K$ (depending on $r_{\varpi}, R_{\varpi}$ and $n$ ), such that $K S_{i}$ covers the slice of $\Omega$ between heights $z_{i}$ and $z_{i}+\ell\left(S_{i}\right)$, for all $i$. Thence, (2-5) follows.

The last thing to prove, then, is that uniformity properties (2-6) and (2-7) hold for every restricted cusp. We use the following result stated by Smith, Stanoyevitch and Stegenga:

Lemma 8.3 [Smith et al. 1994]. Let $\Omega_{1}$ and $\Omega_{2}$ be uniform domains with finite diameters. Then $\Omega_{1} \times \Omega_{2}$ is a uniform domain.

Remark 8.4. The definition of uniform domain used in [Smith et al. 1994] (for the proof of this lemma) is slightly different than the one used here. For the equivalence between the two see [Väisälä 1988] and [Martio 1980].

In Definition 2.6, Properties (2-6) and (2-7) are required for points in $\Omega_{i} \cup \Omega_{i+1}$. We prove that they hold in every slice between heights $z-C \varphi(z)$ and $z+C \varphi(z)$, for every fixed constant $C$. Our proof is based on the following idea: since $\varphi$ is Lipschitz, $\Omega \cap\left\{(x, z) \in \mathbb{R}^{n}: z \in\left(z_{0}-C \varphi\left(z_{0}\right), z_{0}+C \varphi\left(z_{0}\right)\right)\right\}$ is almost the cylinder

$$
\widehat{\Omega}_{0}:=\varphi\left(z_{0}\right) \varpi \times\left(z_{0}-C \varphi\left(z_{0}\right), z_{0}+C \varphi\left(z_{0}\right)\right),
$$

which is uniform thanks to Lemma 8.3. In that lemma, the $\varepsilon$ parameter of $\Omega_{1} \times \Omega_{2}$ depends on the respective values of the parameters of $\Omega_{1}$ and $\Omega_{2}$ and on the quotient $\operatorname{diam} \Omega_{1} / \operatorname{diam} \Omega_{2}$. Since $\operatorname{diam} \Omega_{1} \sim \operatorname{diam} \Omega_{2}$ in (8-2), we may assume that the same $\varepsilon$ holds for the cylinder for every $z_{0}$.

Let $z_{0}>0$ be a fixed number and $C_{0}$ a constant such that $C_{0}<z_{0} / \varphi\left(z_{0}\right)$. Observe that since $t / \varphi(t) \rightarrow \infty$ as $t \rightarrow 0$, the constant $C_{0}$ chosen for a certain $z_{0}$ remains useful for every $z<z_{0}$. Let us set

$$
\Omega_{0}=\Omega \cap\left\{(x, z) \in \Omega: z_{0}-C_{0} \varphi\left(z_{0}\right)<z<z_{0}+C_{0} \varphi\left(z_{0}\right)\right\} .
$$


We want to prove that $\Omega_{0}$ is uniform. We associate points in $\widehat{\Omega}_{0}$ with points in $\Omega_{0}$ at the same heights, so we denote by $(\widehat{x}, z)$ the points in $\widehat{\Omega}_{0}$ and by $(x, z)$ those in $\Omega_{0}$. Let $F: \widehat{\Omega}_{0} \rightarrow \Omega_{0}$ be the function

$$
F(\widehat{x}, z)=\left(\frac{\varphi(z)}{\varphi\left(z_{0}\right)} \widehat{x}, z\right) .
$$

Suppose that $\zeta \in \varpi$ is such that $\varphi\left(z_{0}\right) \zeta=\widehat{x}$. Then $x=\varphi(z) / \varphi\left(z_{0}\right) \widehat{x}=\varphi(z) \zeta$, and $F(\widehat{x}, z)=(x, z) \in \Omega_{0} . F$ is obviously bijective, with

$$
F^{-1}(x, z)=\left(\frac{\varphi\left(z_{0}\right)}{\varphi(z)} x, z\right) .
$$

Now we prove that both $F$ and $F^{-1}$ are Lipschitz with constants independent of $z_{0}$ (this, in turn, shows that $\Omega_{0}$ is uniform). We show only the case $F^{-1}$ since the proof for $F$ is similar. Let us consider $(x, z),(y, w) \in \Omega_{0}, x=\varphi(z) \zeta, y=\varphi(w) \xi$ for some $\zeta, \xi \in \varpi$. We have

$$
\begin{aligned}
\left|F^{-1}(x, z)-F^{-1}(y, w)\right| & =\left|\left(\varphi\left(z_{0}\right) \zeta-\varphi\left(z_{0}\right) \xi, z-w\right)\right| \\
& \leq\left|\varphi\left(z_{0}\right) \zeta-\varphi\left(z_{0}\right) \xi\right|+|x-w|=: \mathrm{I}+\mathrm{II},
\end{aligned}
$$

and

$$
\mathrm{I} \leq \varphi\left(z_{0}\right)\left|\frac{\varphi(z)}{\varphi(z)} \zeta-\frac{\varphi(w)}{\varphi(w)} \xi\right|=\varphi\left(z_{0}\right)\left|\frac{\varphi(w) x-\varphi(z) y}{\varphi(z) \varphi(w)}\right| .
$$

Since $z, w \in\left(z_{0}-C \varphi\left(z_{0}\right), z_{0}+C \varphi\left(z_{0}\right),(8-1)\right.$ implies that $\varphi\left(z_{0}\right) \sim \varphi(z)$, so

$$
\mathrm{I} \leq C\left|\frac{\varphi(w) x-\varphi(z) y}{\varphi(w)}\right| .
$$

On the other hand,

$$
\begin{aligned}
|\varphi(w) x-\varphi(z) y| & \leq|\varphi(w) x-\varphi(w) y|+|\varphi(w) y-\varphi(z) y| \\
& \leq \varphi(w)|x-y|+|\varphi(w)-\varphi(z)||y| \\
& \leq \varphi(w)|x-y|+C_{\varphi}|w-z||\varphi(w) \xi| \\
& \leq C_{\varphi} C_{\varpi} \varphi(w)\{|x-y|+|w-z|\} .
\end{aligned}
$$

Hence $\mathrm{I} \leq C\{|x-y|+|w-z|\}$, and consequently

$$
\left|F^{-1}(x, z)-F^{-1}(y, w)\right| \leq C\{|x-y|+|w-z|\} \leq C|(x, z)-(y, w)| .
$$

So $F^{-1}$ is Lipschitz with a Lipschitz constant depending only on the constants $C_{0}$, $C_{\varphi}$ and $C_{\varpi}$.

Remark 8.5. We do not really need $\Omega_{0}$ to be uniform as a separate domain (with its floor and its roof as parts of the boundary), we just need to prove that the curve joining two points in $\Omega_{0}$ satisfies property (2-7), which is given in terms of the 
distance to the boundary of $\Omega$. But $d_{\partial \Omega}(x, z) \geq d_{\partial \Omega_{0}}(x, z)$, for all $(x, z) \in \Omega_{0}$, so (2-7) holds.

This completes the proof of Claim 8.2. Since the class of domains given by Definition 8.1 is broader than that of Definition A, we can state:

Corollary 8.6. Every domain satisfying Definition A is an external cusp in terms of Definition 2.7 (or Definition 2.6).

Finally, let us observe that items (c) in Theorems 4.1 and 5.1 follow by using the same arguments given in [Maz'ya and Poborchi 1997, pp. 274 and 295], with $\ell(S(z))$ in the role of $\varphi(z)$.

\section{References}

[Acosta and Armentano 2011] G. Acosta and M. G. Armentano, "Finite element approximations in a non-Lipschitz domain: Part II", Math. Comp. 80:276 (2011), 1949-1978. MR 2012i:65248 Zbl pre05962700

[Acosta et al. 2006] G. Acosta, R. G. Durán, and A. L. Lombardi, "Weighted Poincaré and Korn inequalities for Hölder $\alpha$ domains", Math. Methods Appl. Sci. 29:4 (2006), 387-400. MR 2006i:26020 Zbl 1087.26009

[Acosta et al. 2007] G. Acosta, M. G. Armentano, R. G. Durán, and A. L. Lombardi, "Finite element approximations in a nonLipschitz domain", SIAM J. Numer. Anal. 45:1 (2007), 277-295. MR 2009a:65302 Zbl 1140.65079

[Acosta et al. 2012] G. Acosta, R. G. Durán, and F. L. García, "Korn inequality and divergence operator: counterexamples and optimality of weighted estimates", Proc. Amer. Math. Soc. (2012). PII S 0002-9939(2012)11408-X.

[Adams and Fournier 2003] R. A. Adams and J. J. F. Fournier, Sobolev spaces, 2nd ed., Pure and Applied Mathematics 140, Elsevier/Academic Press, Amsterdam, 2003. MR 2009e:46025 Zbl 1098.46001

[Calderón 1961] A.-P. Calderón, “Lebesgue spaces of differentiable functions and distributions”, pp. 33-49 in Proc. Sympos. Pure Math., Vol. IV, edited by C. B. Morrey Jr., American Mathematical Society, Providence, R.I., 1961. MR 26 \#603 Zbl 0195.41103

[Chua 1992] S.-K. Chua, "Extension theorems on weighted Sobolev spaces", Indiana Univ. Math. J. 41:4 (1992), 1027-1076. MR 94a:46035 Zbl 0767.46025

[Chua 1994] S.-K. Chua, "Some remarks on extension theorems for weighted Sobolev spaces", Illinois J. Math. 38:1 (1994), 95-126. MR 94m:46057 Zbl 0797.46026

[Durán and López García 2010a] R. G. Durán and F. López García, "Solutions of the divergence and analysis of the Stokes equations in planar Hölder- $\alpha$ domains", Math. Models Methods Appl. Sci. 20:1 (2010), 95-120. MR 2011h:35219 Zbl 1217.26027

[Durán and López García 2010b] R. G. Durán and F. López García, "Solutions of the divergence and Korn inequalities on domains with an external cusp", Ann. Acad. Sci. Fenn. Math. 35:2 (2010), 421-438. MR 2011k:35180 Zbl 1210.35178

[Jones 1981] P. W. Jones, "Quasiconformal mappings and extendability of functions in Sobolev spaces”, Acta Math. 147:1-2 (1981), 71-88. MR 83i:30014 Zbl 0489.30017

[Kufner 1985] A. Kufner, Weighted Sobolev spaces, John Wiley \& Sons, New York, 1985. Translated from the Czech. MR 86m:46033 Zbl 0567.46009 
[Martio 1980] O. Martio, "Definitions for uniform domains", Ann. Acad. Sci. Fenn. Ser. A I Math. 5:1 (1980), 197-205. MR 82c:30028 Zbl 0469.30017

[Maz'ya 2011] V. Maz'ya, Sobolev spaces with applications to elliptic partial differential equations, 2nd. augmented ed., Grundlehren der Mathematischen Wissenschaften 342, Springer, Heidelberg, 2011. MR 2012a:46056 Zbl 1217.46002

[Maz'ya and Poborchi 1997] V. G. Maz'ya and S. V. Poborchi, Differentiable functions on bad domains, World Scientific Publishing Co., River Edge, NJ, 1997. MR 99k:46057 Zbl 0918.46033

[Smith et al. 1994] W. Smith, A. Stanoyevitch, and D. A. Stegenga, "Smooth approximation of Sobolev functions on planar domains", J. London Math. Soc. (2) 49:2 (1994), 309-330. MR 95e: 46043 Zbl 0797.46029

[Stein 1970] E. M. Stein, Singular integrals and differentiability properties of functions, Princeton Mathematical Series 30, Princeton University Press, 1970. MR 44 \#7280 Zbl 0207.13501

[Väisälä 1988] J. Väisälä, “Uniform domains”, Tohoku Math. J. (2) 40:1 (1988), 101-118. MR 89d: 30027 Zbl 0627.30017

Received February 13, 2012.

GABRIEL ACOSTA

DEPTARTMENT OF MATHEMATICS

UNIVERSITY OF BUENOS AIRES

CIUDAD UNIVERSITARIA

PABELLÓN I

C1428EGA

Buenos Aires

ARGENTINA

gacosta@dm.uba.ar

IGNACIO OJEA

DEPTARTMENT OF MATHEMATICS

UNIVERSITY OF BUENOS AIRES

Ciudad Universitaria

PABELLÓN I

C1428EGA

Buenos Aires

ARGENTINA

iojea@dm.uba.ar 


\title{
PACIFIC JOURNAL OF MATHEMATICS
}

\author{
http://pacificmath.org \\ Founded in 1951 by \\ E. F. Beckenbach (1906-1982) and F. Wolf (1904-1989)
}

\section{EDITORS}

V. S. Varadarajan (Managing Editor)

Department of Mathematics

University of California

Los Angeles, CA 90095-1555

pacific@math.ucla.edu

Vyjayanthi Chari

Department of Mathematics

University of California

Riverside, CA 92521-0135

chari@math.ucr.edu

\section{Robert Finn}

Department of Mathematics Stanford University

Stanford, CA 94305-2125

finn@math.stanford.edu

Kefeng Liu

Department of Mathematics

University of California

Los Angeles, CA 90095-1555

liu@math.ucla.edu
Darren Long

Department of Mathematics

University of California

Santa Barbara, CA 93106-3080

long@math.ucsb.edu

Jiang-Hua Lu

Department of Mathematics

The University of Hong Kong

Pokfulam Rd., Hong Kong jhlu@maths.hku.hk

Alexander Merkurjev

Department of Mathematics

University of California

Los Angeles, CA 90095-1555

merkurev@math.ucla.edu
Sorin Popa

Department of Mathematics University of California

Los Angeles, CA 90095-1555 popa@math.ucla.edu

Jie Qing

Department of Mathematics

University of California

Santa Cruz, CA 95064

qing@cats.ucsc.edu

Jonathan Rogawski

Department of Mathematics

University of California

Los Angeles, CA 90095-1555

jonr@math.ucla.edu

\section{PRODUCTION}

pacific@math.berkeley.edu

\section{SUPPORTING INSTITUTIONS}

ACADEMIA SINICA, TAIPEI

CALIFORNIA INST. OF TECHNOLOGY INST. DE MATEMÁTICA PURA E APLICADA KEIO UNIVERSITY

MATH. SCIENCES RESEARCH INSTITUTE NEW MEXICO STATE UNIV.

OREGON STATE UNIV.

\author{
STANFORD UNIVERSITY \\ UNIV. OF BRITISH COLUMBIA \\ UNIV. OF CALIFORNIA, BERKELEY \\ UNIV. OF CALIFORNIA, DAVIS \\ UNIV. OF CALIFORNIA, LOS ANGELES \\ UNIV. OF CALIFORNIA, RIVERSIDE \\ UNIV. OF CALIFORNIA, SAN DIEGO \\ UNIV. OF CALIF., SANTA BARBARA
}

\author{
UNIV. OF CALIF., SANTA CRUZ \\ UNIV. OF MONTANA \\ UNIV. OF OREGON \\ UNIV. OF SOUTHERN CALIFORNIA \\ UNIV. OF UTAH \\ UNIV. OF WASHINGTON \\ WASHINGTON STATE UNIVERSITY
}

These supporting institutions contribute to the cost of publication of this Journal, but they are not owners or publishers and have no responsibility for its contents or policies.

See inside back cover or pacificmath.org for submission instructions.

The subscription price for 2012 is US \$420/year for the electronic version, and \$485/year for print and electronic.

Subscriptions, requests for back issues from the last three years and changes of subscribers address should be sent to Pacific Journal of Mathematics, P.O. Box 4163, Berkeley, CA 94704-0163, U.S.A. Prior back issues are obtainable from Periodicals Service Company, 11 Main Street, Germantown, NY 12526-5635. The Pacific Journal of Mathematics is indexed by Mathematical Reviews, Zentralblatt MATH, PASCAL CNRS Index, Referativnyi Zhurnal, Current Mathematical Publications and the Science Citation Index.

The Pacific Journal of Mathematics (ISSN 0030-8730) at the University of California, c/o Department of Mathematics, 969 Evans Hall, Berkeley, CA 94720-3840, is published monthly except July and August. Periodical rate postage paid at Berkeley, CA 94704, and additional mailing offices. POSTMASTER: send address changes to Pacific Journal of Mathematics, P.O. Box 4163, Berkeley, CA 94704-0163.

PJM peer review and production are managed by EditFLOW ${ }^{\mathrm{TM}}$ from Mathematical Sciences Publishers.

PUBLISHED BY PACIFIC JOURNAL OF MATHEMATICS

at the University of California, Berkeley 94720-3840

A NON-PROFIT CORPORATION

Typeset in LATEX

Copyright $(02012$ by Pacific Journal of Mathematics 


\title{
PACIFIC JOURNAL OF MATHEMATICS
}

\author{
Volume $259 \quad$ No. $1 \quad$ September 2012
}

Extension Theorems for external cusps with minimal regularity

GABRIEL ACOSTA and IGNACIO OJEA

Convergence of axially symmetric volume-preserving mean curvature flow

MARIA ATHANASSENAS and SEVVANDi KANDANAARACHCHI

On the horoboundary and the geometry of rays of negatively curved manifolds

FranÇOISE DAL'bo, Marc PEIGNÉ and ANDREA

SAMBUSETTI

Two infinite versions of the nonlinear Dvoretzky theorem

KEI FUNANO

Nonlocal uniform algebras on three-manifolds

ALEXANDER J. IZZO

Mahlo cardinals and the torsion product of primary abelian groups

PATRICK W. KEEF

Geometry of trinomials

AARON MELMAN

Drinfeld orbifold algebras

ANNE V. SHEPLER and SARAH Witherspoon

Semi-topological cycle theory I

JYH-HAUR TEH

New construction of fundamental domains for certain Mostow groups 209

TiEHONG ZHAO 